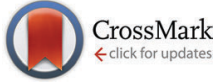

Cite this: Phys. Chem. Chem. Phys., 2016, 18, 7903

Received 10th December 2015 Accepted 2nd February 2016

DOI: $10.1039 / c 5 c p 07634$

www.rsc.org/pccp

\section{Deactivation pathways of thiophene and oligothiophenes: internal conversion versus intersystem crossing $\dagger$}

\begin{abstract}
Patrick Kölle, Thomas Schnappinger and Regina de Vivie-Riedle*
Oligothiophenes and polythiophenes are building blocks of organic-based energy conversion materials. Therefore the lifetime of the excited states plays a central role. As a first step to understand the factors influencing the performance, we investigated the deactivation processes from the first excited state $S_{1}$ of thiophene and small oligothiophenes containing up to four rings using quantum chemical calculations. For thiophene a low-lying $S_{1} / S_{0}$ conical intersection seam is easily accessible and drives the fast internal conversion. In oligothiophenes barriers inhibit this passage while deactivation pathways via intersystem crossing channels open. The first one is responsible for the high triplet quantum yields and takes place shortly after the Franck-Condon region. The second one occurs in the vicinity of a local $\mathrm{S}_{1}$ minimum. The calculated spin-orbit coupling strength together with the singlet-triplet energy gaps can explain the decreasing triplet and increasing fluorescence quantum yields for growing chain length. From the triplets the ground state is reachable by inter-ring torsions and $T_{1} / S_{0}$ intersections. The present results allow a deeper understanding of the deactivation pathways of thiophene and small oligothiophenes and are of potential interest for the photophysics of longer oligothiophenes and polythiophenes used in optical devices.
\end{abstract}

\section{Introduction}

In the last few decades polythiophenes and oligothiophenes have been shown to be some of the most promising candidates of organic materials for technological applications. ${ }^{1,2}$ In particular, they have been used in solar cells, ${ }^{3,4}$ light emitting diodes, ${ }^{5,6}$ photoswitches ${ }^{7}$ and as biological labels. ${ }^{8-10}$ Gaining detailed knowledge of the radiative and nonradiative mechanisms and the factors tuning these processes in the isolated oligomers should be the first step in improving the performance of oligothiophene-based devices.

Static absorption measurements and time-resolved spectroscopic studies have been reported for thiophene (1T) and oligothiophenes containing up to seven thiophene rings. ${ }^{11-40}$ In our nomenclature $n \mathrm{~T}$ is an oligothiophene with $n$ thiophene units. The monomer $1 \mathrm{~T}$ was shown to be non-fluorescent and nonphosphorescent. ${ }^{37}$ With the aid of static quantum chemical ${ }^{41-43}$ and nonadiabatic molecular dynamics calculations ${ }^{44-46}$ it was revealed that $1 \mathrm{~T}$ decays ultrafast primarily via its singlet states and conical intersections to the ground state without the

Department of Chemistry, Ludwig-Maximilians-Universität München,

Butenandtstraße 5-13, 81377 München, Germany.

E-mail: Regina.de_Vivie-Riedle@cup.uni-muenchen.de

$\dagger$ Electronic supplementary information (ESI) available: Complete computational results and optimized geometries: Tables S1-S20 and Fig. S1-S23. See DOI: $10.1039 / \mathrm{c} 5 \mathrm{cp} 07634 \mathrm{j}$ involvement of triplet states. In contrast the rates of internal conversion (IC) of the oligothiophenes are very small and the relaxation processes are dominated by triplet formation and fluorescence. ${ }^{13,21,22,25}$ It was shown that the fluorescence quantum yields increase while the triplet quantum yields decrease when extending the chain length of the oligomer. These trends are mainly attributed to changes in the nonradiative decay processes, which are dominated by effective intersystem crossing (ISC) from the singlet to the triplet manifold. Photodetachment photoelectron spectroscopy (PD-PES) measurements ${ }^{32,47}$ explained this dependence of the triplet quantum yield as a function of the oligomer size mainly by the growing energy differences between the singlet $\mathrm{S}_{1}$ state and the triplet $\mathrm{T}_{2}$ state. It is important to note that the triplet energies determined by PD-PES are based on radical anions. The anion equilibrium structure of the oligothiophenes is mostly planar and thus closer to the minimum structure of the $S_{1}$ states than to the non-planar minimum of the neutral ground states. ${ }^{47}$ Therefore the triplet energies and the state order deduced from PD-PES are also not related to the FranckCondon (FC) region but to the structure of the $\mathrm{S}_{1}$ minimum. For bithiophene (2T) it was shown by quantum chemical calculations that the triplet $T_{4}$ and $T_{3}$ states are below the $S_{1}$ state in the nonplanar $S_{0}$ minimum geometry but above the $S_{1}$ state in its planar equilibrium geometry. ${ }^{48}$ Furthermore femtosecond time-resolved spectroscopy measurements suggested that for $2 \mathrm{~T}$ and $3 \mathrm{~T}$ ultrafast ISC takes place from a twisted $\mathrm{S}_{1}$ state responsible for the highly 
effective triplet generation..$^{28,33,34}$ In addition oligothiophenes are considered to be quite flexible molecules with respect to the rotation around the inter-ring bonds. Nevertheless the $\mathrm{T}_{4}$ and $\mathrm{T}_{3}$ states were regarded until now to play only a minor role in the efficient ISC pathways of the oligothiophenes.

In this work, we will demonstrate the crucial role of the $\mathrm{T}_{3}$ state for the effective triplet formation and elucidate the details of the ISC processes and relaxation pathways of bithiophene (2T), terthiophene (3T) and quaterthiophene (4T). By quantum chemical calculations of the low-lying excited states and the spin-orbit coupling between the $S_{1}$ and the triplet states we will show that efficient and ultrafast ISC occurs along the geometry relaxation of the $S_{1}$ state leading to the high triplet quantum yields of the oligothiophenes. In addition we will reveal why the relaxation pathway of thiophene is dominated by its singlet states and conical intersections (CoIns) and why these CoIns are not active anymore in the oligothiophenes. The current results in conjunction with previous work offer quite a complete picture of the photophysical properties of the molecules and may be linked to the application of the oligothiophenes in optical devices.

\section{Computational details}

The ground state optimizations of $1 \mathrm{~T}-4 \mathrm{~T}$ have been carried out using the B3LYP functional ${ }^{49-52}$ and the $6-311 G^{* *}$ basis set. $^{53}$ In a theoretical study of $2 \mathrm{~T}$ it was demonstrated that the optimized geometries at the B3LYP/6-311G** level of theory exhibit the best agreement with the experiments and that the torsional angle between the adjacent thiophene rings is very sensitive to both the basis set and the method used. ${ }^{54}$

The electronic states of $1 \mathrm{~T}, 2 \mathrm{~T}$ and $3 \mathrm{~T}$ were computed with the complete active space second-order perturbation theory method (CASPT2), ${ }^{55}$ the equation of motion coupled cluster singles and doubles method (CCSD) ${ }^{56}$ and the time-dependent density functional theory method (TDDFT) using the CAM-B3LYP functional. ${ }^{57}$ The electronic states of the larger system $4 \mathrm{~T}$ were calculated using the CCSD and the TDDFT methods. For the CCSD calculations the $6-311+G^{* *}(1 T-3 T)$ and $6-31 G^{*}(4 T)$ basis sets ${ }^{58}$ were used. The TDDFT calculations were carried out using the $6-311+G^{* *}$ basis set, while for the more demanding CASPT2 method the $6-31 \mathrm{G}^{*}$ basis set was used throughout.

The reference wave function and the molecular orbitals for the CASPT2 calculation were determined using the state-averaged complete active space self-consistent field method (SA-CASSCF). For $1 \mathrm{~T}$ and $3 \mathrm{~T}$ five singlet and four triplet states were included in the state-averaging procedure while for $2 \mathrm{~T}$ six singlet and four triplet states were incorporated. The CASPT2 calculations were performed using a shift of 0.3 a.u. For $1 \mathrm{~T}$ the active space was composed of eight electrons and seven orbitals (CAS(8/7)). In addition to the $\pi$-orbitals one pair of $\sigma$-orbitals $\left(\sigma / \sigma^{*}\right)$ was included in the active space. The geometry optimizations of the excited states of 1T have been carried out at the CASPT2 level of theory. The Hessian matrix for the optimization of the transition state of the $S_{1}$ state was calculated numerically.
The active space of $2 \mathrm{~T}$ contained one $\sigma^{*}$-orbital in addition to the $\pi$-space (12 electrons/10 orbitals) in all calculations to describe the $\pi \sigma^{*}$ singlet state $(\operatorname{CAS}(12 / 11))$. For the calculation of the $S_{1} / S_{0}$ conical intersections, the $T_{1} / S_{0}$ intersection and the $S_{1}\left(\pi \sigma^{*}\right)$ minima either one (CAS(14/12)) or two pairs $(\operatorname{CAS}(16 / 14))$ of $\sigma$-orbitals were included in the active space depending on whether one or two $\mathrm{C}-\mathrm{S}$ bond cleavages should be described. For the calculation of the spin-orbit coupling matrix elements (SOMEs) along the relaxation path from the FC point to the local $S_{1}\left(\pi \pi^{*}\right)$ minimum the CAS(12/11) active space was used. The SOME computations along the ring-opening path leading to one broken C-S bond were performed with the CAS(14/12) active space. The optimizations of the excited states, conical intersections and the $T_{1} / S_{0}$ intersection of $2 \mathrm{~T}$ have been carried out using the CASSCF method.

For the energy calculations of $3 \mathrm{~T}$ the complete $\pi$-valence active space was used (CAS(18/15)). Like in a previous study of $3 \mathrm{~T}^{59}$ the CASSCF optimizations of the excited states were performed with a smaller active space, 12 electrons/12 orbitals, where the three deepest $\pi$-orbitals were kept inactive. For the optimization of the $S_{1} / S_{0}$ conical intersection and the $T_{1} / S_{0}$ intersection of $3 \mathrm{~T}$ this active space was extended by one pair of $\sigma$-orbitals $\left(\sigma / \sigma^{*}\right)$. For the SOME calculations along the relaxation path from the FC point to the local $S_{1}\left(\pi \pi^{*}\right)$ minimum the smaller $\pi$-active space $(\operatorname{CAS}(12 / 12))$ was extended by three $\sigma^{*}$-orbitals (CAS(12/15)) to keep the active space stable. For $4 \mathrm{~T}$ the optimizations of the excited states have been carried at the TDDFT/6-31G* level.

The spin-orbit coupling (SOC) strength between selected singlet $\left(\mathrm{S}_{1}\right)$ and triplet $\left(\mathrm{T}_{k}\right)$ states was computed as

$$
\mathrm{SOC}_{\mathrm{lk}}=\sqrt{\sum_{u}\left|\left\langle\mathrm{~T}_{1, u}\left|\hat{H}_{\mathrm{SO}}\right| \mathrm{S}_{k}\right\rangle\right|^{2}} \quad u=x, y, z
$$

which can be considered as the length of the spin-orbit coupling vector SOC $_{\mathrm{lk} \cdot}{ }^{60}$ Its component $\left\langle\mathrm{T}_{\mathrm{l}, u}\left|\hat{H}_{\mathrm{SO}}\right| \mathrm{S}_{k}\right\rangle$ corresponds to the calculated SOME. The SOMEs were calculated by an efficient method using the Breit-Pauli spin-orbit operator ${ }^{61}$ implemented in the Molpro software package. ${ }^{62,63}$

The CASSCF method has been employed to compute the transition dipole moments. Energy differences corrected by the CASPT2 method were used in the oscillator strength formula. The program package MOLPRO ${ }^{62,63}$ was used for the CASSCF and CASPT2 calculations, while the DFT, TDDFT and CCSD calculations were carried out using the Gaussian 09 software package. ${ }^{64}$

\section{Results and discussion}

\subsection{Vertical excited states of thiophene (1T) and oligothiophenes (2T-4T)}

The optimized structures for the ground state minima of 1T-4T are shown in Fig. 1. The $1 \mathrm{~T}$ geometry is planar and has $C_{2 \mathrm{v}}$ symmetry, while all others are non-planar. The trans conformation of $2 \mathrm{~T}$ exhibits $C_{2}$ symmetry and is characterized by a torsional angle $\alpha$ of $150.4^{\circ}$ between the rings. This conformation has been shown to be the global minimum of the internal rotational 

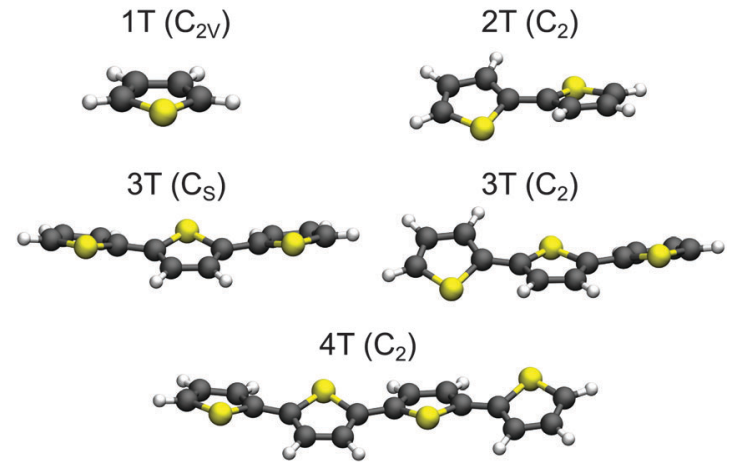

Fig. 1 Optimized B3LYP/6-311G** ground state geometries of $1 T-4 T$. For $3 T$ both nearly isoenergetic minima are shown. The point groups of the structures are given in parentheses.

potential surface. ${ }^{54}$ For $3 \mathrm{~T}$ two nearly isoenergetic minima exist among the total number of ten already reported in previous theoretical studies. ${ }^{59,65-67}$ The global minimum is the twisted trans-trans-syn conformation with $C_{\mathrm{s}}$ symmetry $\left(\alpha=153.8^{\circ}\right)$ and the trans-trans-anti conformation with $C_{2}$ symmetry $\left(\alpha=154.6^{\circ}\right)$ is only $0.02 \mathrm{eV}(\Delta G)$ above. Also the transition state between both conformers lies only $0.05 \mathrm{eV}(\Delta G)$ above the global minimum (Fig. S13 and Table S10 in the ESI $\dagger$ ). The flatness of the torsional potential is in agreement with the experimental observation that more than one twisted conformation is present in solution. ${ }^{68}$ The all-trans conformation found as a minimum structure with $C_{2}$ symmetry for $4 \mathrm{~T}$ is characterized by an $\alpha$ value of $157.1^{\circ}$ between the central rings. Like a previous study ${ }^{47}$ our calculations show that the degree of planarity increases from $2 \mathrm{~T}$ $\left(\alpha=150^{\circ}\right)$ to $4 \mathrm{~T}\left(\alpha=157^{\circ}\right)$.

At these geometries the low-lying $\pi \pi^{*}$ and the $\pi \sigma^{*}$ singlet states plus the first four triplet states of $1 \mathrm{~T}-4 \mathrm{~T}$ were computed. The excitation energies, the electronic characters and oscillator strengths are listed in Table 1 together with the corresponding experimental data. To facilitate the comparison of the electronic states of the different systems, the HOMO of all molecules is denoted $\pi_{1}$ and the LUMO $\pi_{1}^{*}$ orbital (for details, see ESI $\dagger$ ). When possible the calculations were performed on the CASPT2, CCSD and TDDFT levels of theory for comparison and we found a good agreement for all molecules studied. A complete list with the results of all methods used is given in Tables S1-S5 in the ESI. $\dagger$ Our focus is on the bright $S_{1}$ state and its relaxation paths after optical excitation as this state is discussed to be crucial for the use of oligothiophenes in organic devices. The $\pi \sigma^{*}$ singlet state is responsible for a nonradiative relaxation via a ring opening path and the triplet states induce the ISC. The results in Table 1 are discussed from these aspects. In the FC region the $S_{1}$ state has $\pi \pi^{*}$ character and is completely delocalized for all investigated systems. The excitation energy of the $\mathrm{S}_{1}$ state decreases from $1 \mathrm{~T}$ to $4 \mathrm{~T}$ and its corresponding oscillator strength increases. The calculated vertical excitation energy of the $S_{1}$ state agrees very well for $1 \mathrm{~T}$ and $2 \mathrm{~T}$ with the experimental values while for $3 \mathrm{~T}$ and $4 \mathrm{~T}$ larger deviations are observed. The use of larger basis sets reduces these deviations (see Tables S12, S13 and S16, ESI $\dagger$ ). But the combination with the large active spaces required for $3 \mathrm{~T}$ and $4 \mathrm{~T}$ (see Section 3.2.4) cannot be handled within a reasonable computation time. The state order of the $\pi \sigma^{*}$ singlet state increases systematically from the $S_{3}$ state in $1 T$ to the $S_{7}$ state in $4 \mathrm{~T}$ as more and more $\pi \pi^{*}$ states intrude (see Tables S1-S5 in the ESI $\dagger$ ). The $\pi \sigma^{*}$ state is due to the $\sigma^{*}$ character more localized and does not profit as much as the $\pi \pi^{*}$ states from the elongation of the $\pi$-system.

In $1 \mathrm{~T}$ the $\mathrm{T}_{3}$ and $\mathrm{T}_{4}$ states have $\pi \sigma^{*}$ character and lie substantially above the $S_{1}$ state. The energy gap to the $T_{2}$ state with $\pi \pi^{*}$ character is also large $(0.83 \mathrm{eV})$. In $2 \mathrm{~T}$ more $\pi \pi^{*}$ states exist due to the extension of the system. $\mathrm{T}_{2}$ and $\mathrm{T}_{3}$ are such additional $\pi \pi^{*}$ states and lie below the $S_{1}$ state with a small $S_{1}-T_{3}$ energy gap of only $0.10 \mathrm{eV}$ (see Table 1). The $\mathrm{T}_{4}$ state has comparable character to the $\mathrm{T}_{2}$ state of $1 \mathrm{~T}$ and is nearly isoenergetic with the $\mathrm{S}_{1}$ state in agreement with a previous study. ${ }^{48}$ Also in the larger oligothiophenes the four lowest triplet states are characterized

Table 1 Calculated vertical singlet and triplet excitation energies (eV) for the low-lying valence excited states plus the $\pi \sigma^{*}$ singlet state of $1 T-4 T$ at their ground-state minima compared with experimental data. For $3 T$ the excitation energies are shown for both isoenergetic conformers $\left(C_{s}\right.$ and $C_{2}$ symmetry). Oscillator strengths are given in parentheses. The HOMO of all molecules is denoted $\pi_{1}$ and the LUMO $\pi_{1}{ }^{*}$ orbital (for details, see ESI)

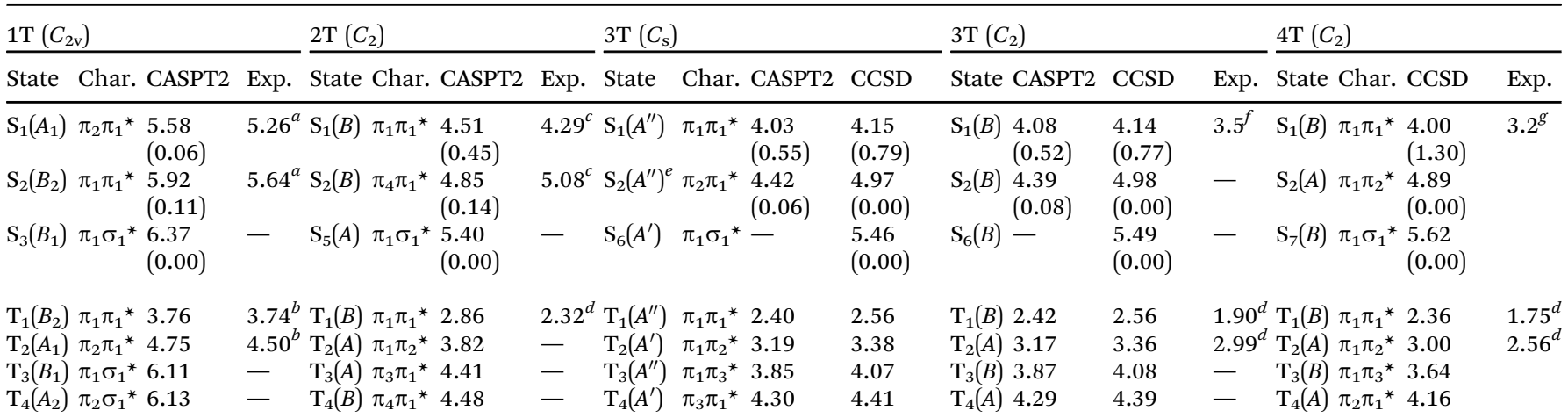

${ }^{a}$ Magnetic circular dichroism. ${ }^{11 b}$ Electron energy loss spectroscopy. ${ }^{36}{ }^{c}$ Gas-phase absorption spectrum at room temperature. ${ }^{19 d}$ Photodetachment photoelectron spectrum in the gas phase. ${ }^{4}{ }^{e}$ At the CASPT2 level of theory the $\mathrm{S}_{2}$ state of both the $3 \mathrm{~T}$ conformers is described by an $\pi_{2} \pi_{1}{ }^{*}$ excitation, while with the CCSD method the $\mathrm{S}_{2}$ state has $\pi_{1} \pi_{2}{ }^{*}$ character and $A^{\prime}$ and $A$ symmetry, respectively, (see Tables S3 and S4 in the ESI). ${ }^{f}$ Absorption spectrum in solution at room temperature. ${ }^{22,25,30} \mathrm{~g}$ Absorption spectrum in solution at room temperature. ${ }^{13,22,25}$ 
by $\pi \pi^{*}$ excitations, whereby the $\mathrm{T}_{4}$ state becomes more localized for $3 \mathrm{~T}$ and $4 \mathrm{~T}$ and therewith shifts slightly above the $\mathrm{S}_{1}$ state. In $3 \mathrm{~T}$ the $\mathrm{S}_{1}$ state is energetically close to the $\mathrm{T}_{3}$ state. At the CASPT2 level of theory only the inclusion of all $\pi$-orbitals in the active space results in the correct electronic state order consistent with the CCSD results. The use of a smaller active space leads to artificial stabilization of the $S_{1}$ state with respect to the triplet states shifting it below the $\mathrm{T}_{3}$ state $^{59}$ (see Table S11 in the ESI $\dagger$ ). Also in $4 \mathrm{~T}$ the $\mathrm{S}_{1}$ state is only slightly above the $\mathrm{T}_{3}$ state at the CCSD level of theory.

Of the studied molecules only $1 \mathrm{~T}$ exhibits large singlet-triplet energy gaps at the FC point. In combination with the low-lying $\pi \sigma^{*}$ singlet state $S_{3}$ this gives a first hint why photoexcited $1 \mathrm{~T}$ decays primarily via singlet states and no intersystem crossing occurs. ${ }^{37,41-43}$ In contrast thereto, the oligothiophenes $2 \mathrm{~T}-4 \mathrm{~T}$ show small singlet-triplet energy gaps, which however increase with the chain length $\left(0.03 \mathrm{eV}\right.$ for $2 \mathrm{~T}, 0.18 \mathrm{eV}\left(C_{\mathrm{s}}\right)$ and $0.21 \mathrm{eV}\left(C_{2}\right)$ for $3 \mathrm{~T}$ and $0.36 \mathrm{eV}$ for $4 \mathrm{~T})$. This is consistent with the experimental observation of extremely high triplet quantum yields for the oligothiophenes, which however decrease again with size ( 0.99 for $2 \mathrm{~T}, 0.95$ for $3 \mathrm{~T}$ and 0.73 for $4 \mathrm{~T}){ }^{25}$ The details of the relaxation pathways of the different systems will be discussed in the next sections.

\subsection{Deactivation pathways}

For 1T-3T all results are at the CASPT2 level of theory and when possible confirmed by CCSD calculations. For the larger $4 \mathrm{~T}$ system the CCSD and TDDFT methods were used.

3.2.1 Thiophene (1T). For thiophene it was demonstrated by theory ${ }^{41-44}$ and experiment ${ }^{37}$ that after excitation to the $S_{1}\left(\pi \pi^{*}\right)$ state, it quickly relaxes to the local $S_{1}$ minimum $\left(S_{1}\right.$-Min-a, Fig. 2). This process is described by a time constant of $80 \mathrm{fs}^{37}$ Thereafter the main part of the population overcomes a barrier to reach the global minimum of the $S_{1}$ state $\left(S_{1}-M i n-b\right)$. This relaxation is characterized by a ring-opening via the dissociative $\pi \sigma^{*}$ character and a time constant of 25 fs leading to C-S bond

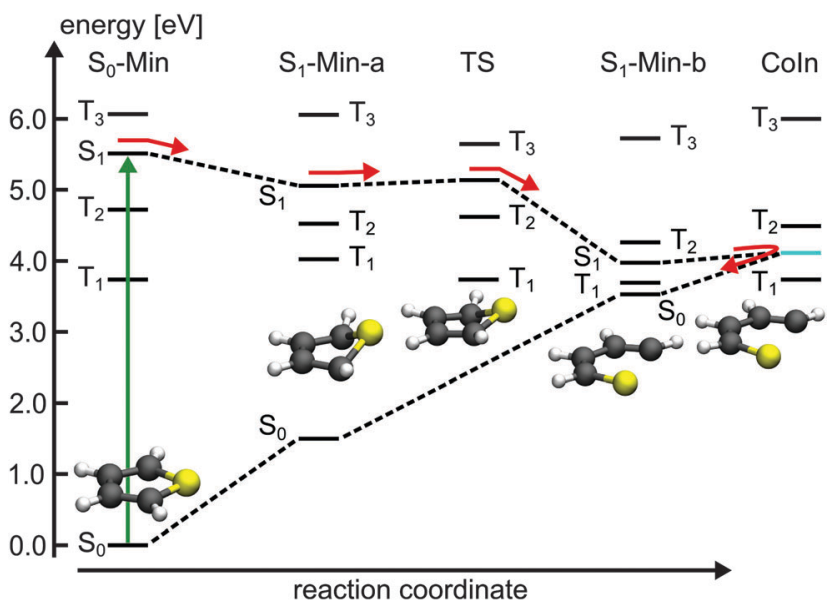

Fig. 2 Schematic illustration of the proposed deactivation mechanism of $1 T$ after excitation to the $S_{1}$ state. The deactivation pathway is represented by red arrows. The $S_{1} / S_{0}$ Coln is indicated by cyan line. The relevant optimized geometries are shown as well. cleavage. In the vicinity of $\mathrm{S}_{1}$-Min-b, a conical intersection (CoIn) with the ground state, associated with a small barrier of $0.06 \mathrm{eV},{ }^{43}$ completes the ultrafast internal conversion.

So far the barrier height for the ring-opening from $\mathrm{S}_{1}$-Min-a to $\mathrm{S}_{1}$-Min-b was estimated by linear interpolation at the CCSD level of theory to be $0.26 \mathrm{eV}$ and understood as a consequence of an avoided crossing. ${ }^{43}$ We were able to locate the transition state (TS) separating the two $\mathrm{S}_{1}$ minima at the CASPT2 level of theory (Fig. 2, for details, see Fig. S2 and Table S6 in the ESI $\dagger$ ). Therewith the barrier height is reduced to $0.07 \mathrm{eV}$. We also found that the CoIn is part of a low lying and thus very efficient seam along the $d_{\text {SCCC }}$ dihedral angle (see Fig. S3 and S4 in the ESI $\dagger$ ).

Salzmann et al. ${ }^{41}$ argued that due to vanishing spin-orbit coupling (SOC) and large $\mathrm{S}_{1}$-triplet energy gaps at the FC point and at $\mathrm{S}_{1}$-Min-a (see Fig. 2) a notable probability for ISC may only exist along the ring-opening path. However the ISC has to compete with the highly effective irreversible ring-opening path through the CoIn seam. Thus our additional results further support the interpretation that thiophene after excitation to the $\mathrm{S}_{1}$ state decays mainly via its singlet electronic states, which is consistent with the observed dynamics and the absence of fluorescence and phosphorescence. ${ }^{37}$

\subsubsection{Bithiophene (2T)}

Triplet states and intersystem crossing. In $2 \mathrm{~T}$ the triplet states $\mathrm{T}_{4}$ and $\mathrm{T}_{3}$ are below and close to the $\mathrm{S}_{1}$ state at the FC point. Optimization of the $S_{1}$ state leads to the conversion of the nonplanar structure to a planar minimum with $C_{2 \mathrm{~h}}$ symmetry $\left(\mathrm{S}_{1}\right.$-Min-a) and lowers its energy below the $\mathrm{T}_{4}$ and $\mathrm{T}_{3}$ states (see Table 2). We constructed a simplified reaction path $\mathrm{RC}_{\mathrm{S}}$ by linear interpolation between the optimized $\mathrm{S}_{0}$-Min and $\mathrm{S}_{1}$-Min-a geometries. The calculated energies along the $\mathrm{RC}_{\mathrm{S}}$ reveal the intersection of the $S_{1}$ state with the $T_{4}$ and $T_{3}$ states (Fig. 3a).

For an effective ISC between singlet and triplet states, the spin-orbit coupling (SOC) should be reasonably strong and the states involved should be close in energy. First semiempirical calculations using the INDO/SCI approach showed for 2T, 3T and $6 \mathrm{~T}$ that the SOC values decay with decreasing torsion angle $\alpha^{69}$

Table 2 Calculated CASPT2 vertical singlet and triplet excitation energies (eV) for the low-lying valence excited states of $2 \mathrm{~T}$ at the ground-state $\left(S_{0}-M i n\right)$ and $S_{1}$ state $\left(S_{1}-\right.$ Min-a) minima. In addition the lowest excited singlet state with $\pi \sigma^{\star}$ character is given $\left(S_{5}\right)$. Oscillator strengths are shown in parentheses

\begin{tabular}{|c|c|c|c|c|c|c|}
\hline \multirow[b]{2}{*}{ State } & \multirow[b]{2}{*}{ Character } & \multicolumn{2}{|c|}{$\underline{\mathrm{S}_{0}-\operatorname{Min}\left(C_{2}\right)}$} & \multicolumn{3}{|c|}{$\underline{\mathrm{S}_{1} \text {-Min-a }\left(C_{2 \mathrm{~h}}\right)}$} \\
\hline & & Sym. & CASPT2 & Sym. & CASPT2 & Exp. \\
\hline$S_{1}$ & $\pi_{1} \rightarrow \pi_{1}^{*}$ & $B$ & $4.51(0.45)$ & $B_{\mathrm{u}}$ & $3.71(0.43)$ & $3.43^{a}$ \\
\hline$S_{2}$ & $\pi_{4} \rightarrow \pi_{1}^{*}$ & $B$ & $4.85(0.14)$ & $A_{\mathrm{g}}$ & $4.22(0.00)$ & - \\
\hline $\mathrm{S}_{5}$ & $\pi_{1} \rightarrow \sigma_{1}^{*}$ & $A$ & $5.40(0.00)$ & $A_{\mathrm{u}}^{\mathrm{s}}$ & $4.74(0.00)$ & - \\
\hline $\mathrm{T}_{1}$ & $\pi_{1} \rightarrow \pi_{1}^{*}$ & $B$ & 2.86 & $B_{\mathrm{u}}$ & 1.99 & $2.32^{b}$ \\
\hline $\mathrm{T}_{2}$ & $\pi_{1} \rightarrow \pi_{2}^{*}$ & $A$ & 3.82 & $A_{\mathrm{g}}$ & 3.57 & - \\
\hline $\mathrm{T}_{3}$ & $\pi_{3} \rightarrow \pi_{1}^{*}$ & $A$ & 4.41 & $A_{\mathrm{g}}^{\mathrm{s}}$ & 4.11 & - \\
\hline $\mathrm{T}_{4}$ & $\pi_{4} \rightarrow \pi_{1}^{*}$ & $B$ & 4.48 & $B_{\mathrm{u}}$ & 4.26 & - \\
\hline
\end{tabular}

${ }^{a}$ Maximum of the fluorescence spectrum in dioxane at room temperature. ${ }^{25}$ ${ }^{b}$ Photodetachment photoelectron spectrum in the gas phase. ${ }^{47}$ 

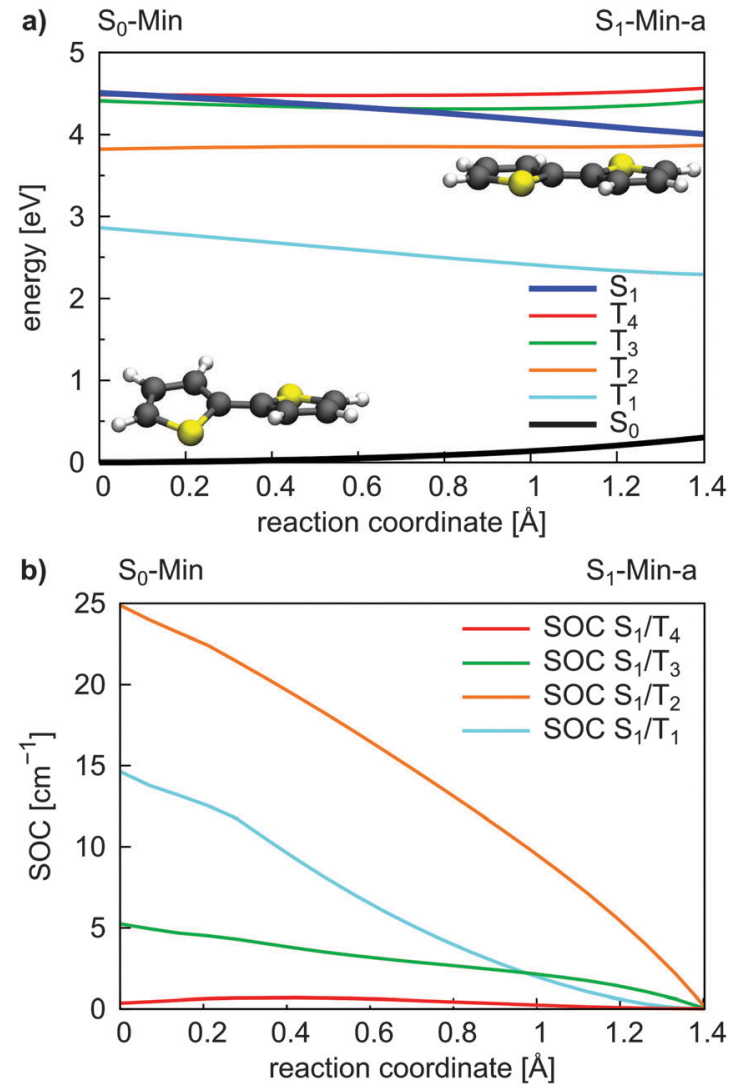

Fig. 3 Energy profile of the $S_{0}$, the $S_{1}$ and the four lowest lying triplet states (a) and the SOC between the $\mathrm{S}_{1}$ and triplet states (b) along the $\mathrm{S}_{1}$ relaxation coordinate of $2 \mathrm{~T}$. The energy calculations were performed at the CASPT2/6-31G* level of theory and confirmed at the CCSD level (Fig. S9 in the ESI $\dagger$ ). The SOCs were calculated using the CASSCF method. The reaction coordinate was generated by linear interpolation between the optimized $\mathrm{S}_{0}$-Min and $\mathrm{S}_{1}$-Min-a geometries.

Since we are interested in the relaxation pathways from the $S_{1}$ state, we calculated the SOC between the $S_{1}$ and the four lowest lying triplet states along the reaction coordinate $\mathrm{RC}_{\mathrm{S}}$ at the CASSCF level (Fig. 3b).

All SOC values also decrease along this coordinate and vanish at $S_{1}$-Min-a. Between $S_{1}$ and $T_{2} / T_{3}$ the SOC is zero for symmetry reasons, between $S_{1}$ and $T_{1} / T_{4}$ it vanishes, although the coupling is symmetry allowed. In general, for strong SOC, contributions from orbitals of heavy atoms have to be involved in the electronic configuration of the mixing states. The $\sigma^{*}$ orbital is localized on the $S$-atom and occurs via small $\pi \sigma^{*}$ contributions in the $S_{1}$ state of $2 \mathrm{~T}$. This $\pi \sigma^{*}$ contribution systematically decays along the $\mathrm{RC}_{\mathrm{S}}$ as the planarization reduces the $\sigma / \pi$ mixing. Correspondingly, all SOCs, also the symmetry allowed ones, decrease and vanish along this coordinate.

In view of the calculated SOC and the $\mathrm{S}_{1}$-triplet energy gaps, the highest ISC probability along the relaxation coordinate $\mathrm{RC}_{\mathrm{S}}$ exists with the $T_{3}$ state. The intermediate degeneracy of the $S_{1}$ and the $\mathrm{T}_{3}$ states should compensate for their moderate spin-orbit interaction and allow a fast ISC. An example for such a scenario has been shown recently for uracil by Richter et al. ${ }^{70}$ and has been discussed for benzene by Worth and co-workers. ${ }^{71,72}$

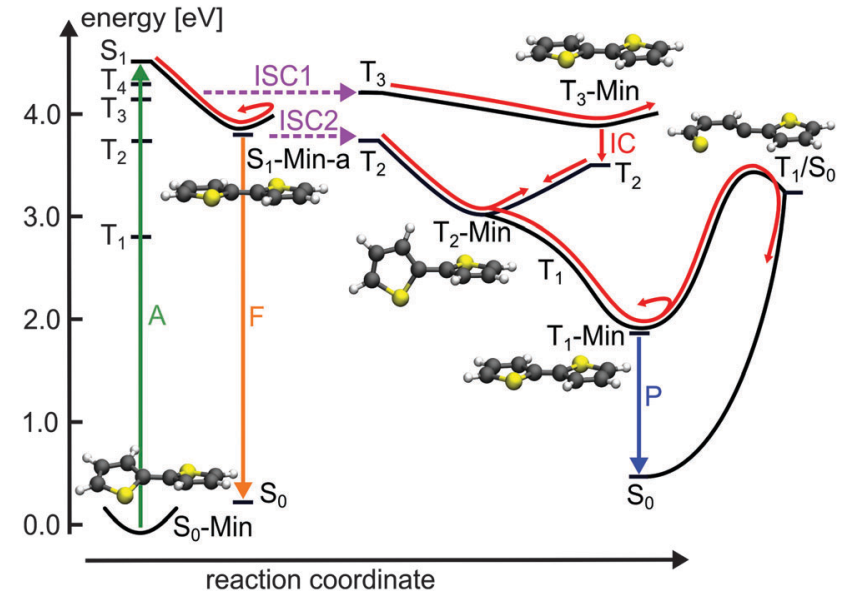

Fig. 4 Schematic illustration of the proposed deactivation mechanism of $2 \mathrm{~T}$ after excitation to the $\mathrm{S}_{1}$ state (A: absorption; $\mathrm{F}$ : fluorescence; ISC: intersystem crossing; IC: internal conversion; $\mathrm{P}$ : phosphorescence). The deactivation pathway is represented by red arrows. The relevant optimized geometries are shown as well.

For the population that reaches $\mathrm{S}_{1}$-Min-a a second ISC path opens between the $S_{1}$ and the $T_{2}$ states (ISC2, see Fig. 4). Here both states are close in energy and although the SOC vanishes at the minimum geometry, the torsional out-of-plane motion can induce moderate coupling. Such motions have been shown to be highly active during the $\mathrm{S}_{1}$ dynamics of $2 \mathrm{~T}-4 \mathrm{~T}^{45,46}$

The possibility of two ISC channels is consistent with the experimental observations of Paa et al. ${ }^{34}$ using femtosecond time-resolved spectroscopy. They obtained a biexponential decay for the transient $S_{1} \rightarrow S_{n}$ absorption (ESA) bands, with a fast ( $\tau=1.4 \mathrm{ps})$ and slow $(\tau=29.6 \mathrm{ps})$ component. In addition the rise of the triplet-triplet absorption (TTA) is characterized by a fast time constant of $1.4 \mathrm{ps}$ and a slower one of $58 \mathrm{ps}$. Therefore the authors proposed an ISC mechanism with two channels: the fast one starting from a twisted $S_{1}$ state geometry and taking place during the initial geometry relaxation. The second slower ISC channel starts from the relaxed $S_{1}$ state and competes with fluorescence from $\mathrm{S}_{1}$-Min-a. Although the oscillator strength is reasonable at $S_{1}$-Min-a (see Table 2), the fluorescence quantum yield is reported to be small $\left(\phi_{\mathrm{F}}=0.014\right) .{ }^{25}$ Thus we suggest that the major part of the $\mathrm{S}_{1}$ population decays via the two ISC paths. The quantum yield $\phi_{\mathrm{F}}$ of $2 \mathrm{~T}$ is increased when the temperature is lowered from 298 to $77 \mathrm{~K}^{25}$ Under these conditions the first ISC path is less populated as the lower temperature enhances the planarity of the ground state. ${ }^{25}$

Next we discuss the relaxation from $T_{3}$ and $T_{2}$ back to the ground state. The $T_{3}$ relaxation leads to the planar $T_{3}$ state minimum where the $T_{3}$ and $T_{2}$ states are close in energy $(\Delta E=0.28 \mathrm{eV}$, see Table S8 in the ESI $\dagger)$. Therewith the $\mathrm{T}_{2}$ state can also be populated by a fast internal conversion from $\mathrm{T}_{3}$ (Fig. 4). $\mathrm{T}_{2}$ is the only state considered which is described by an excitation to the $\pi_{2}{ }^{*}$ orbital (see Table 2). This orbital is characterized by an antibonding $\pi$-interaction between the thiophene rings (see Fig. S5 in the ESI $\dagger$ ), therefore the $\mathrm{T}_{2}$ minimum is a twisted structure with orthogonal thiophene rings ( $\mathrm{T}_{2}$-Min, Fig. 4). 
At this minimum the $T_{2}$ state is degenerate with the $T_{1}$ state $^{47}$ (see Table S8 in the ESI $\dagger$ ) suggesting the existence of a $\mathrm{T}_{2} / \mathrm{T}_{1}$ CoIn from where relaxation into the global excited state minimum $\mathrm{T}_{1}$-Min (Fig. 4) can take place. The calculated vertical emission energy at this planar minimum is $1.77 \mathrm{eV}$ and corresponds to the maximum of the phosphorescence spectrum $(2.06 \mathrm{eV}) .^{32}$ Since the phosphorescence is weak $\left(\phi_{\mathrm{P}}=10^{-5}\right),{ }^{32}$ we conclude that by far most of the population return back to the ground state by a nonradiative process. ${ }^{25}$ This process will be discussed in combination with a possible decay via CoIns in the next section.

Conical intersections. A remaining question is why for $2 \mathrm{~T}$ the deactivation path is dominated by ISC not by the ring-opening path via the $\mathrm{S}_{1} / \mathrm{S}_{0}$ CoIns which is the major path for $1 \mathrm{~T}$. We therefore optimized the $S_{1} / S_{0}$ CoIns and the $S_{1}$-Min-b minima for $2 \mathrm{~T}$. For $1 \mathrm{~T}$ only one open-chain structure with a broken $\mathrm{C}-\mathrm{S}$ bond is distinguishable. For the trans conformation of $2 \mathrm{~T}$ several open-chain structures are possible. First of all four singly and six doubly opened geometries can be discriminated. The singly opened structures can be planar or non-planar and the inner or the outer $\mathrm{C}-\mathrm{S}$ bond can be broken. The four optimized CoIns are shown in Fig. 5 (CoIn1-4) and are as in $1 T$ in the vicinity of the $S_{1}-$ Min-b minima. In all these structures the $S_{1}$ state has $\pi \sigma^{*}$ character while the $S_{0}$ state is the closed shell electronic configuration. The geometries of the CoIns1-4 and the $\mathrm{S}_{1}$-Min-b minima are quite similar to the corresponding ones of $1 \mathrm{~T}$, e.g. the distances of the broken $\mathrm{C}-\mathrm{S}$ bonds are nearly identical (1T CoIn: $3.41 \AA$ ). Also the barrier from the $S_{1}-M i n-b$ minima to the CoIns is at most $0.1 \mathrm{eV}$ (Table 3) and mainly associated with a small elongation of the C-S bond (see Fig. S10 in the ESI $\dagger$ ). CoIn 1 and CoIn 3 lie below and CoIn2 and CoIn4 above $\mathrm{S}_{1}$-Min-a (see Table 3 ). The cleavage of an outer C-S bond leads to steric repulsion between the sulfur atom and the adjacent thiophene ring (see Fig. 5) resulting in the destabilization of CoIn2 and CoIn4. The cleavage of the inner $\mathrm{C}-\mathrm{S}$ bonds
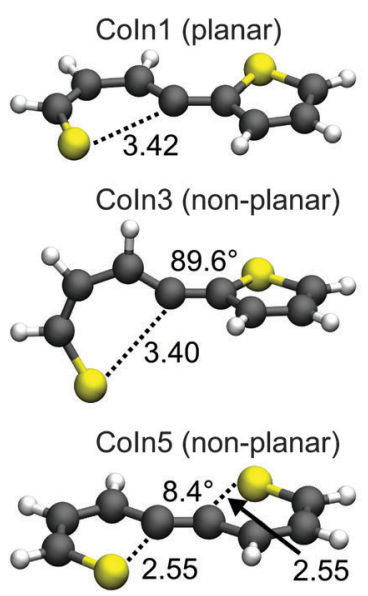

Fig. 5 Optimized geometries of the conical intersections (Colns) and the $\mathrm{T}_{1} / \mathrm{S}_{0}$ intersection of $2 \mathrm{~T}$ obtained at the CASSCF/6-31G* level of theory. The distance of the broken $\mathrm{C}-\mathrm{S}$ bonds are given in Angstrom $(\AA)$. For the non-planar geometries the torsional angle between the thiophene rings is also shown (defined by the $\mathrm{S}-\mathrm{C}-\mathrm{C}-\mathrm{S}$ dihedral angle).
Table 3 Adiabatic CASPT2 excitation energies (eV) to the $S_{1}-$ Min-b minima and the optimized Colns. The stabilization energies of the Colns relative to the $S_{1}-$ Min-a are given. Negative values indicate destabilization. The barriers between $S_{1}$-Min- $a$ and $S_{1}$-Min-b were obtained by linear interpolation between the optimized geometries and calculated at the CCSD/6-31G* level of theory

\begin{tabular}{lcrrrr}
\hline & CoIn1 & CoIn2 & CoIn3 & CoIn4 & CoIn5 \\
\hline S $_{1}$-Min-b & 3.75 & 4.13 & 3.58 & 4.12 & - \\
Conical intersection & 3.85 & 4.22 & 3.60 & 4.20 & 4.09 \\
Stabilization energy & 0.16 & -0.21 & 0.41 & -0.19 & -0.08 \\
Barrier & 0.40 & 0.87 & 1.04 & 0.92 & - \\
\hline
\end{tabular}

leads to a lower steric repulsion and explains the stabilization of CoIn $1(0.2 \mathrm{eV})$ and CoIn3 $(0.4 \mathrm{eV})$, whereby CoIn3 is the lowest intersection due to the orthogonal position of the thiophene rings.

Based on these results we identified the most promising one of the six doubly opened geometries. The optimized non-planar structure CoIn5 (Fig. 5) shows that when two C-S bonds are broken the $S_{1} / S_{0}$ degeneracy is achieved already for smaller elongations of the C-S distance to $2.55 \AA$ A. Nevertheless CoIn5 lies above $S_{1}$-Min-a (Table 3 ) and is further neglected.

From the energetics at least CoIn1 and CoIn3 could be reached from $\mathrm{S}_{1}$-Min-a. But the possible barriers in between have to be considered. This was investigated again by using simplified reaction paths $\mathrm{RC}_{\mathrm{S} 2}$ constructed by linear interpolation between $\mathrm{S}_{1}$-Min-a, $\mathrm{S}_{1}$-Min-b and the respective CoIn. Based on the good agreement between CASPT2 and CCSD results obtained for the energy profile along the $\mathrm{RC}_{\mathrm{S}}$ (see Fig. 3a and Fig. S9 in the ESI $\dagger$ ), we calculated the four continuing ringopening paths $\mathrm{RC}_{\mathrm{S} 2}$ using the faster CCSD method. The singlereference method CCSD has previously been used to study the deactivation paths of thiophene ${ }^{43}$ and furan ${ }^{73}$ and was found to give energies of good quality even in the vicinity of CoIns. This is also found for the paths towards CoIn1/CoIn3 (Fig. 6) and CoIn2/CoIn4 (Fig. S11 in ESI $\dagger$ ). The positions of the CoIns at the CCSD level are only slightly shifted with respect to the CASSCF results and thus the CCSD results are sufficiently accurate to estimate the barriers. The reported values for the barriers in Table 3 are an upper limit for the real ones. Optimization of the corresponding transition states would lower these barriers as shown for $1 \mathrm{~T}$ (see Section 3.2.1). Nevertheless from the barrier heights one can see that CoIn1 is associated with the smallest barrier of $0.4 \mathrm{eV}$ and the lowest CoIn (CoIn3) with a barrier of $1.0 \mathrm{eV}$. This can be understood as the geometrical distortion necessary to reach the non-planar CoIn3 from the planar $\mathrm{S}_{1}$-Min-a is significantly larger than from the planar CoIn1. This is also reflected in the larger $\mathrm{RC}_{\mathrm{S} 2}$ values for CoIn3 (Fig. 6b).

In summary, CoIn 1 is the most favorable intersection of $2 \mathrm{~T}$ and should be reachable due to an accumulated relaxation energy of $0.5 \mathrm{eV}$ from the FC point to $\mathrm{S}_{1}$-Min-a. Thus some of the excited molecules should decay to the ground state through this passage. This is, however, in contrast to the measured nearunity triplet quantum yield of $2 \mathrm{~T}$. A possible explanation is that also along the ring-opening path to CoIn 1 the $S_{1}$ state can couple to the triplet states. We calculated the four lowest lying triplet states along this path and, indeed found several intersections 

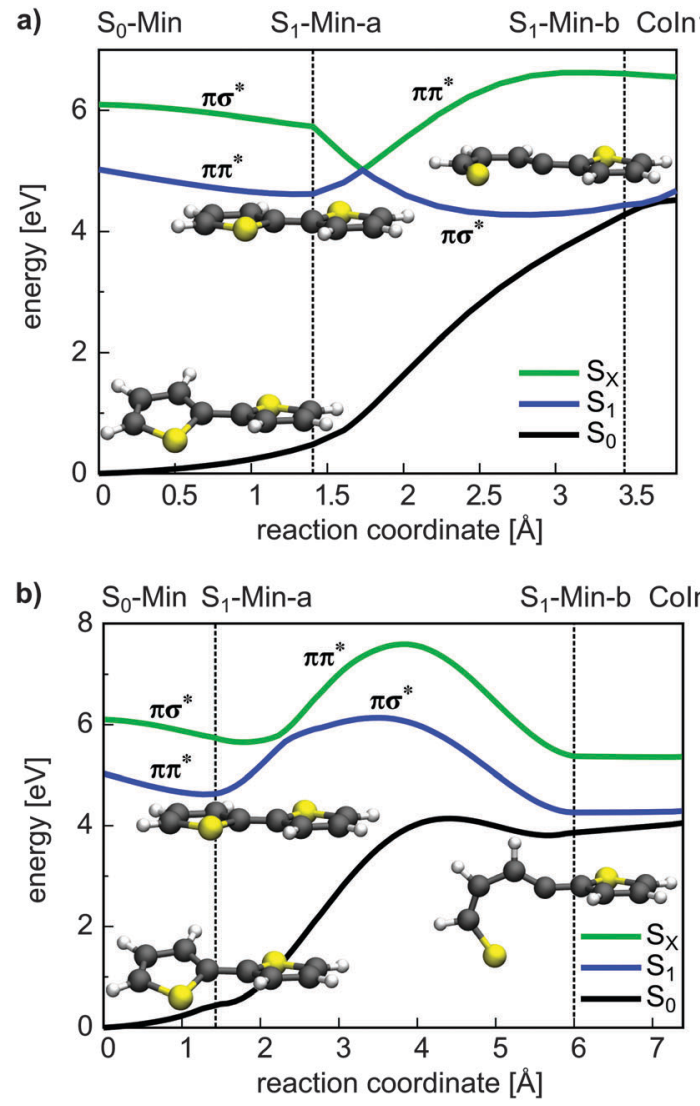

Fig. 6 Energetic course of the ground, $\pi \pi^{*}$ and $\pi \sigma^{*}$ singlet states along the reaction path leading to Coln1 (a) and Coln3 (b). The energy calculations were performed at the CCSD/6-31G* level of theory. The reaction coordinates were generated by linear interpolation between the CASSCF optimized geometries. The positions of the minima and Colns are indicated by vertical lines. The electronic character and the minimum structures are shown as well along each path.

between the $S_{1}$ and the triplet states (Fig. 7). In addition near and after the barrier strong SOC of the $\mathrm{S}_{1}$ state with the triplet states occur $\left(\approx 20-100 \mathrm{~cm}^{-1}\right.$, Table S9 in the ESI $\left.\dagger\right)$ due to the rising $\pi \sigma^{*}$ character of the $S_{1}$ state.

The results clearly elucidate why the deactivation path of $2 \mathrm{~T}$ is dominated by ISC and the relaxation path of $1 \mathrm{~T}$ by $\mathrm{S}_{1} / \mathrm{S}_{0}$ CoIns. First of all after excitation to the $S_{1}$ state only for $2 \mathrm{~T}$ effective ISC possibilities exist during the initial motion (compare Fig. 2 and 3). Secondly the barrier to reach a CoIn is higher for $2 \mathrm{~T}(0.40 \mathrm{eV})$ than for $1 \mathrm{~T}(0.26 \mathrm{eV})$. And finally even if the barrier to the $\mathrm{S}_{1} / \mathrm{S}_{0}$ CoIn is overcome, the ISC to several triplet states is probable in $2 \mathrm{~T}$ and depopulates the $S_{1}$ state.

The calculations along the ring-opening path $\mathrm{RC}_{\mathrm{S} 2}$ (Fig. 7) also reveal the existence of a low lying intersection between the $\mathrm{T}_{1}$ and the $\mathrm{S}_{0}$ state. The optimized $\mathrm{T}_{1} / \mathrm{S}_{0}$ intersection is a planar structure with a broken C-S bond of $3.04 \AA$ (Fig. 5) where the $\mathrm{T}_{1}$ state has $\pi \sigma^{*}$ character and accordingly large SOC values with the $\mathrm{S}_{0}$ state $\left(\approx 100 \mathrm{~cm}^{-1}\right)$. This intersection found along the ringopening path could also be responsible for the overall relaxation from the triplets back to the ground state. We therefore estimated the barrier from the endpoint of the triplet cascade, $\mathrm{T}_{1}-\mathrm{Min}$, to

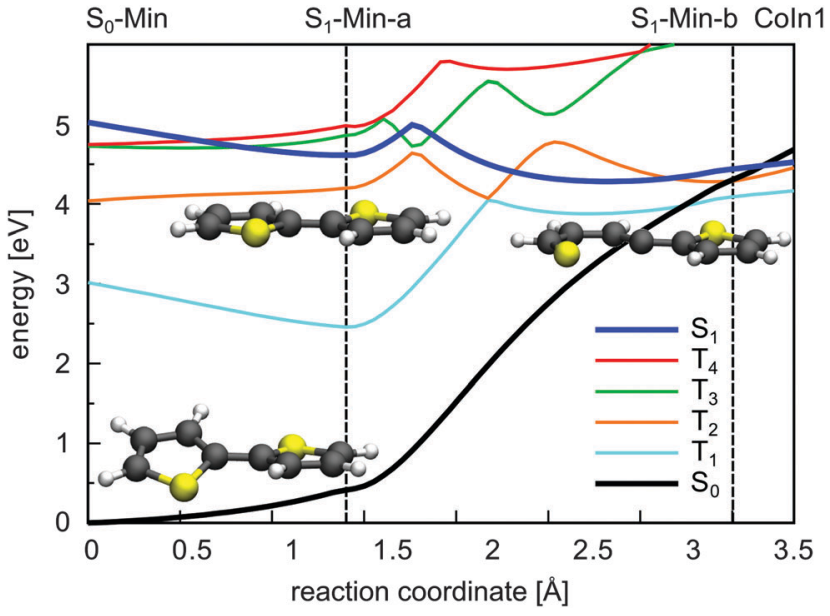

Fig. 7 Adiabatic course of the $S_{0}$, the $S_{1}$ and the four lowest lying triplet states along the reaction path leading to Coln1. The energy calculations were performed at the CCSD/6-31G* level of theory. The reaction coordinate was generated by linear interpolation between the CASSCF optimized geometries. The positions of the minima and Colns are indicated by vertical lines. The minimum structures are shown as well along the path.

the $\mathrm{T}_{1} / \mathrm{S}_{0}$ intersection by linear interpolation at the CASPT2 level of theory (Fig. S12 in the ESI $\dagger$ ). The calculated barrier height of $1.39 \mathrm{eV}$ should account for the long triplet lifetime of $100 \mu$ s of $2 \mathrm{~T}^{25}$ The reaction coordinate of this path is mainly characterized by an elongation of the $\mathrm{C}-\mathrm{S}$ distance. As the gradient difference vector at the $T_{1} / S_{0}$ intersection is nearly orthogonal to this reaction coordinate (Fig. S10, ESI $\dagger$ ), the probability for intersystem crossing is quite high after the system has crossed the barrier (for details, see ESI $\dagger$ ). All in all the $\mathrm{T}_{1} / \mathrm{S}_{0}$ intersection completes the deactivation pathway of $2 \mathrm{~T}$.

3.2.3 Terthiophene (3T). In $3 \mathrm{~T}$ for both nearly isoenergetic ground state minima (Section 3.1) the $\mathrm{T}_{4}$ state is now above and the $\mathrm{T}_{3}$ state is slightly below the bright $\mathrm{S}_{1}$ state (see Table 4). Relaxation of the $S_{1}$ state again leads to a planar minimum $\left(\mathrm{S}_{1}\right.$-Min-a) with $C_{2 \mathrm{v}}$ symmetry. The energy profiles are calculated at the CCSD/6-31G* level along the linear interpolated reaction coordinate $\mathrm{RC}_{\mathrm{S}}$ between the isoenergetic ground state minima and $S_{1}$-Min-a. As in $2 T$ the $S_{1}$ state intersects with the $T_{3}$ state (Fig. 8a and Fig. S19a, ESI $\dagger$ ) and the SOC between the $S_{1}$ and the triplet states decrease along the $\mathrm{RC}_{\mathrm{S}}$ and vanishes at the planar $\mathrm{S}_{1}$-Min-a (Fig. 8b and Fig. S19b, ESI $\dagger$ ). Analogous to $2 \mathrm{~T}$ this can be understood by symmetry selection rules and varying $\pi \sigma^{*}$ contribution in the $S_{1}$ state. In the planar $C_{2 v}$ geometry the spin-orbit coupling between the $\mathrm{S}_{1}\left(B_{1}\right)$ and the $\mathrm{T}_{1} / \mathrm{T}_{3}\left(B_{1}\right)$ is again symmetry forbidden. Overall the $\pi \sigma^{*}$ contribution in the $S_{1}$ state is smaller for $3 \mathrm{~T}$ than for $2 \mathrm{~T}$ resulting in generally lower SOC values. The intermediate degeneracy of the $S_{1}$ state and the $T_{3}$ state should again compensate their moderate spin-orbit interaction making $\mathrm{T}_{3}$ the most probable candidate for an efficient ISC along the initial relaxation (ISC1, see Fig. 9). Like in $2 \mathrm{~T}$ the $\mathrm{T}_{2}$ and the $\mathrm{S}_{1}$ state come closer during geometry relaxation to $\mathrm{S}_{1}$-Min-a. ${ }^{59}$ At the CCSD/6-31G* level the $\mathrm{S}_{1}-\mathrm{T}_{2}$ energy gap is still $0.64 \mathrm{eV}$ (see Table S12 in the ESI $\dagger$ ). But the gap reduces to $0.46 \mathrm{eV}$ using the larger basis set $6-311+\mathrm{G}^{* *}$ and approaches 
Table 4 Calculated CASPT2 vertical singlet and triplet excitation energies (eV) for the low-lying valence excited states of $3 T$ at the ground-state $\left(\mathrm{S}_{0}-\mathrm{Min}\right)$ and the $\mathrm{S}_{1}$ state $\left(\mathrm{S}_{1}-\mathrm{Min}-\mathrm{a}\right)$ minima. Oscillator strengths are shown in parentheses

\begin{tabular}{llllllll}
\hline & & \multicolumn{2}{l}{$\mathrm{S}_{0}$-Min $\left(C_{\mathrm{S}}\right)$} & & \multicolumn{2}{c}{$\mathrm{S}_{1}$-Min-a $\left(C_{2 \mathrm{v}}\right)$} \\
State & Character & Sym. & CASPT2 & & Sym. & CASPT2 & Exp. \\
\hline $\mathrm{S}_{1}$ & $\pi_{1} \rightarrow \pi_{1}{ }^{*}$ & $A^{\prime \prime}$ & $4.03(0.55)$ & $B_{1}$ & $3.08(0.68)$ & $2.91^{a}$ \\
$\mathrm{~S}_{2}$ & $\pi_{2} \rightarrow \pi_{1}{ }^{*}$ & $A^{\prime \prime}$ & $4.42(0.06)$ & $A_{1}{ }^{b}$ & $3.56(0.00)$ & - \\
$\mathrm{T}_{1}$ & $\pi_{1} \rightarrow \pi_{1}{ }^{*}$ & $A^{\prime \prime}$ & 2.40 & $B_{1}$ & 1.75 & $1.90^{c}$ \\
$\mathrm{~T}_{2}$ & $\pi_{1} \rightarrow \pi_{2}{ }^{*}$ & $A^{\prime}$ & 3.19 & & $A_{1}$ & 2.87 & $2.99^{c}$ \\
$\mathrm{~T}_{3}$ & $\pi_{1} \rightarrow \pi_{3}{ }^{*}$ & $A^{\prime \prime}$ & 3.85 & & $B_{1}$ & 3.81 & - \\
$\mathrm{T}_{4}$ & $\pi_{3} \rightarrow \pi_{1}{ }^{*}$ & $A^{\prime}$ & 4.30 & & $A_{1}$ & 4.19 & -
\end{tabular}

${ }^{a}$ Maximum of the fluorescence spectrum in dioxane at room temperature. ${ }^{25 b}$ At $\mathrm{S}_{1}$-Min-a the $\mathrm{S}_{2}$ state is described by an double excitation $\left(\pi_{1} \rightrightarrows \pi_{1}^{*}\right)$. ${ }^{c}$ Photodetachment photoelectron spectrum in the gas phase. ${ }^{47}$
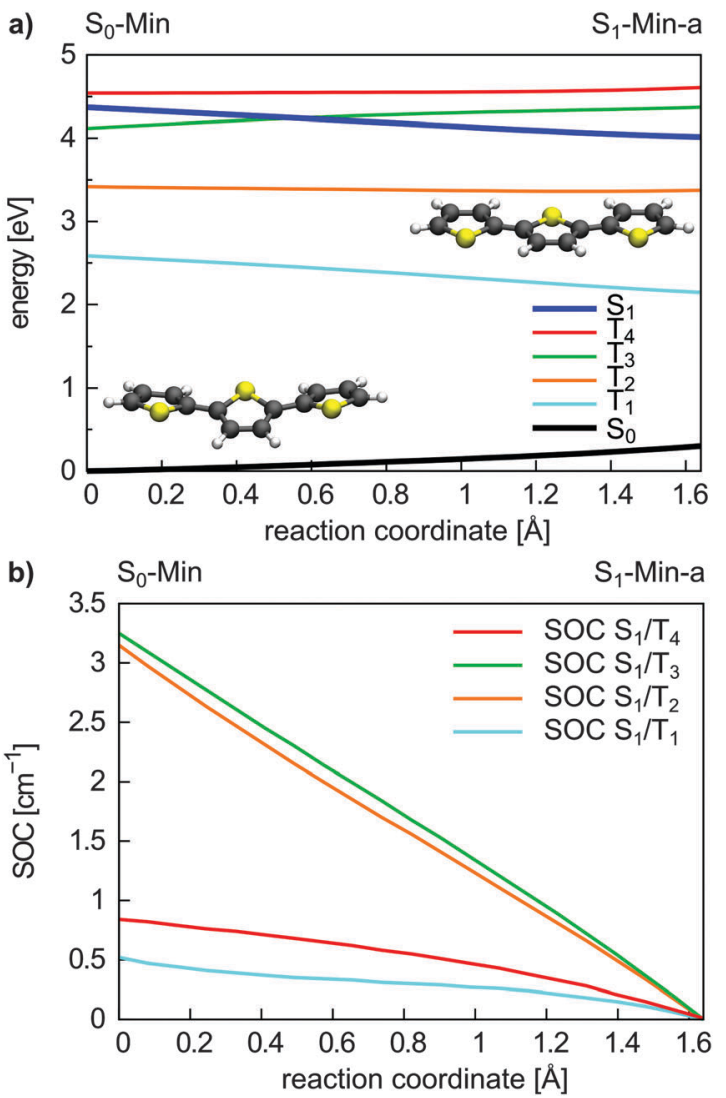

Fig. 8 Energy profile of the $S_{0}$, the $S_{1}$ and the four lowest lying triplet states (a) and SOC between the $S_{1}$ and triplet states (b) along the $S_{1}$ relaxation coordinate of $3 \mathrm{~T}$. The energy calculations were performed at the CCSD/6-31G* level of theory and the SOCs were calculated with the CASSCF method. The reaction coordinate was generated by linear interpolation between the optimized $S_{0}-$ Min $\left(C_{s}\right.$ symmetry $)$ and $S_{1}-$ Min-a $\left(C_{2 v}\right.$ symmetry) geometries.

the value of $0.21 \mathrm{eV}$ obtained at the CASPT2/6-31G* level (Table 4). Analogous to $2 \mathrm{~T}$ for the second ISC process (ISC2, see Fig. 9) out of the $\mathrm{S}_{1}$-Min-a some torsional vibrational activity is needed.

Also for $3 \mathrm{~T}$ both ISC channels are confirmed by experimental results. Beyond that the experimental observations can now be understood more deeply. Rentsch and co-workers carried out

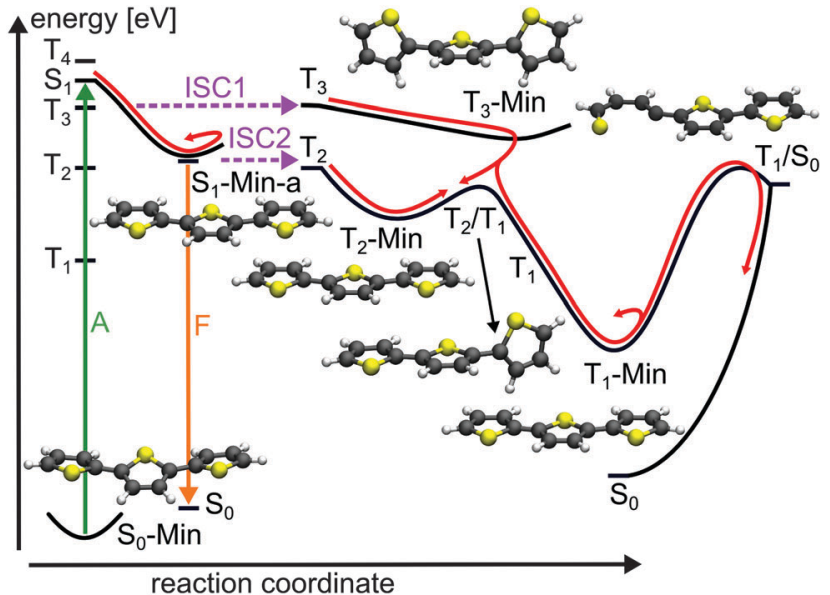

Fig. 9 Schematic illustration of the proposed deactivation mechanism of 3T after optical excitation to the $S_{1}$ state (A: absorption; F: fluorescence; ISC: intersystem crossing). The deactivation pathway is represented by red arrows. The relevant optimized geometries are shown as well.

femtosecond time-resolved spectroscopy with dependence on the excitation wavelength. ${ }^{28,33,34}$ They observed a biexponential decay for the $\mathrm{S}_{1}$ state with a fast and slow component occurring in parallel with the triplet formation. Both processes and the triplet quantum yield were found to depend on the excitation energy. At a wavelength of $400 \mathrm{~nm}$ (low-energy side of the $S_{0} \rightarrow S_{1}$ absorption band) the $S_{1}$ decay and the rise of the TTA are determined by the fluorescence lifetime of the $\mathrm{S}_{1}$ state $(165 \mathrm{ps}) .{ }^{34}$ With increasing excitation energies an additional fast channel for triplet formation with a time constant of about 2 ps occurs. The triplet quantum yield reaches a maximum value at $381 \mathrm{~nm}$ and remains constant up to $370 \mathrm{~nm}$. Rentsch and co-workers suggested that the fast ISC channel is populated by excitation of non-planar molecules and opens while the planar $\mathrm{S}_{1}$-Min-a is approached. With the low energy pulse mainly planar molecules are excited among the ensemble as the planar conformation has the lowest excitation energy. ${ }^{74}$ The fast ISC channel is considered to be responsible for the highly effective triplet formation and has been quantified by temperature dependent measurements of the fluorescence quantum yield by Rossi et al. ${ }^{75}$ They estimated that more than half of the $\mathrm{T}_{1}$ population of $3 \mathrm{~T}$ arises from this channel. The details of the two ISC channels are clearly elucidated by the results of the present work. Only if non-planar conformations are excited the fast ISC from the $S_{1}$ state to the $T_{3}$ state can happen. If planar conformations are excited thermal torsional vibrational activity can induce the second ISC from the $\mathrm{S}_{1}$ to the $\mathrm{T}_{2}$ state.

Like in $2 \mathrm{~T}$ a remaining question is the possible role of $\mathrm{S}_{1} / \mathrm{S}_{0}$ CoIns as deactivation path. Based on the results of $2 \mathrm{~T}$ we focused on the most promising one and optimized a planar $\mathrm{S}_{1} / \mathrm{S}_{0}$ conical intersection with a singly broken $\mathrm{C}-\mathrm{S}$ bond. We also located the corresponding $\mathrm{S}_{1}$ minimum $\left(\mathrm{S}_{1}-\mathrm{Min}-\mathrm{b}\right)$, which was found again in the vicinity of the CoIn. The barrier between $\mathrm{S}_{1}$-Min-a and $\mathrm{S}_{1}$-Min-b was estimated by linear interpolation at the CCSD/6-31G* level of theory (see Fig. S20 in the ESI $\dagger$ ). The optimized CoIn geometry (Fig. 10) is similar to the ones of the 


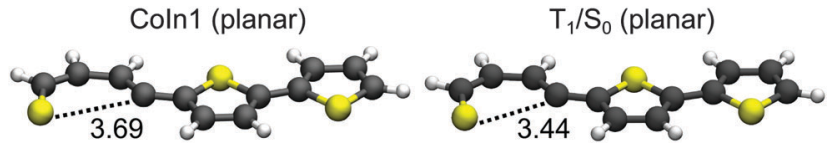

Fig. 10 Optimized geometries of the conical intersection (Coln) and the $\mathrm{T}_{1} / \mathrm{S}_{0}$ intersection of $3 \mathrm{~T}$ obtained at the CASSCF/6-31G* level of theory. The distance of the broken $\mathrm{C}-\mathrm{S}$ bonds are given in Angstrom ( $\mathrm{A})$.

smaller systems, only the C-S distance (3.69 $\AA)$ is slightly larger than in $2 \mathrm{~T}(3.42 \AA)$ and $1 \mathrm{~T}(3.41 \AA)$.

In Table 5 the relevant points of the pathway to the CoIns are compared for $1 \mathrm{~T}-3 \mathrm{~T}$. The adiabatic energy difference in the CoIns stays in the same range while the $S_{1}\left(\pi \pi^{*}\right)$ state is significantly stabilized at the FC point and at $S_{1}$-Min-a due to the increased $\pi$ system. At the CoIns the $S_{1}$ state has $\pi \sigma^{*}$ character and is thus more localized and does not profit to the same extent from the elongation of the $\pi$-system. The significant lowering of the $\pi \pi^{*}$ relative to the $\pi \sigma^{*}$ character in the $S_{1}$ state is also reflected by the increased barrier between $S_{1}$-Min-a and $S_{1}-$ Min-b from $1 T$ to $3 T$ inhibiting the passage to the $S_{1} / S_{0}$ CoIn for the oligothiophenes. The higher barrier for $3 \mathrm{~T}$ relative to $2 \mathrm{~T}$ is confirmed by the even smaller rate constant for internal conversion. ${ }^{25}$ The low barrier for $1 \mathrm{~T}$ can be further understood comparing the structures of the $\mathrm{S}_{1}$-Min-a minima. Only in $1 \mathrm{~T}$ it is non-planar (Fig. 2) and the C-S bond is already slightly elongated in comparison to $\mathrm{S}_{0}$-Min. Thus in $1 \mathrm{~T}$ the initial relaxation is directed towards the ring-opening while the initial relaxation of $2 \mathrm{~T}$ and $3 \mathrm{~T}$ is characterized by interring rotation leading to planar geometries.

From the planar $\mathrm{S}_{1}$-Min-a the ring-opening path is thus highly unlikely for 3T. Even if the high barrier is overcome, several intersections of the $S_{1}$ state with the triplet states (see Fig. S20 in the ESI $\dagger)$ with strong SOC $\left(\approx 20-100 \mathrm{~cm}^{-1}\right.$, Table S15 in the ESI $\dagger$ ) exist and would again induce ISC. Another deactivation possibility out of $\mathrm{S}_{1}$-Min-a is fluorescence. The $\mathrm{S}_{1}$ state has a reasonable oscillator strength and the calculated vertical energy compares well with the maximum of the fluorescence spectrum (see Table 4). The higher fluorescence quantum yield of $3 \mathrm{~T}\left(\phi_{\mathrm{F}}=\right.$ $0.054)$ in comparison to $2 \mathrm{~T}\left(\phi_{\mathrm{F}}=0.024\right)^{25}$ can be explained by the lower SOC values along the initial relaxation towards $\mathrm{S}_{1}$-Min-a and the higher barrier for the ring-opening path. Still the major part of the $\mathrm{S}_{1}$ population decays via ISC.

The depopulation pathways of the triplet states proceeds again via a cascade. Relaxation of the $\mathrm{T}_{3}$ state leads to its minimum.

Table 5 Adiabatic excitation energies (eV) of the $S_{1}$ state at the FC point, $\mathrm{S}_{1}$ minima $\left(\mathrm{S}_{1}-\mathrm{Min}-\mathrm{a}\right.$ and $\left.\mathrm{S}_{1}-\mathrm{Min}-\mathrm{b}\right)$ and at the Coln of $1 \mathrm{~T}, 2 \mathrm{~T}$ (Coln1) and $3 \mathrm{~T}$ at the CASPT2/6-31G* level of theory. The barriers between $\mathrm{S}_{1}$-Min-a and $\mathrm{S}_{1}$-Min- $\mathrm{b}$ were obtained by linear interpolation between the optimized geometries and calculated using the CCSD method. The value for the barrier of $1 T$ is taken from ref. 17

\begin{tabular}{llll}
\hline & $1 \mathrm{~T}$ & $2 \mathrm{~T}$ & $3 \mathrm{~T}$ \\
\hline FC point & 5.58 & 4.51 & 4.03 \\
$\mathrm{~S}_{1}$-Min-a & 5.07 & 4.01 & 3.27 \\
$\mathrm{~S}_{1}$-Min-b & 4.00 & 3.75 & 4.16 \\
Conical intersection & 4.08 & 3.85 & 4.32 \\
Barrier & 0.26 & 0.40 & 0.81
\end{tabular}

Like the $T_{2}$ state of $2 \mathrm{~T}$ the $\mathrm{T}_{3}$ state of $3 \mathrm{~T}$ is described by an excitation to an antibonding $\pi$-orbital $\left(\pi_{3}^{*}\right.$, Table 4$)$ with nodal planes between the thiophene rings (see Fig. S14 in the ESI $\dagger$ ). Accordingly, the $\mathrm{T}_{3}$ minimum is a twisted structure with orthogonal thiophene rings ( $T_{3}$-Min, Fig. 9). At this minimum the first three triplet states are close in energy (see Table S14 in the ESI $\dagger$ ) and a fast internal conversion from $T_{3}$ to $T_{2}$ or $T_{1}$ can happen. The $T_{2}$ minimum is planar ( $T_{2}$-Min, Fig. 9 ) and in contrast to the non-planar $\mathrm{T}_{2}$-Min of $2 \mathrm{~T}$ now an energy gap of 0.76 exists between $T_{2}$ and $T_{1}$ and a barrier of $0.32 \mathrm{eV}$ has to be overcome to reach the $T_{2} / T_{1}$ conical intersection. The $T_{2} / T_{1}$ conical intersection is characterized by one terminal thiophene ring being orthogonal to two planar thiophene rings (Fig. 9). In $T_{1}$ the global excited state minimum is reached, which is again planar (Fig. 9). As no phosphorescence was detected, ${ }^{25}$ a possible relaxation back to the ground state is again via the $\mathrm{T}_{1} / \mathrm{S}_{0}$ intersection with one broken $\mathrm{C}-\mathrm{S}$ bond (Fig. 10). We estimated again the barrier between $\mathrm{T}_{1}$-Min and $\mathrm{T}_{1} / \mathrm{S}_{0}$ by interpolation at the CCSD level of theory. The high value of $2.17 \mathrm{eV}$ to reach this intersection could account for the long triplet lifetime of $3 \mathrm{~T}^{25}$ But after crossing this barrier, the probability for intersystem crossing is quite high like in $2 \mathrm{~T}$ (for details, see ESI $\dagger$ ).

3.2.4 Quaterthiophene (4T). For $3 \mathrm{~T}$ we showed that only the inclusion of all $\pi$-orbitals in the active space of the CASPT2 calculations results in the correct electronic state order of the $\mathrm{S}_{1}$ and the triplet states. This order, especially $\mathrm{T}_{3}$ below $\mathrm{S}_{1}$ at the FC point, proved to be crucial to understand and explain the ISC processes resulting in high triplet quantum yields of $2 \mathrm{~T}$ and $3 \mathrm{~T}$. For $4 \mathrm{~T}$ such an active space means 24 electrons in 20 orbitals. In spite of symmetry restrictions, this active space is too large to be handled within a reasonable computation time. Due to the good agreement of the CCSD and TDDFT results with the CASPT2 results for 1T-3T (see Tables S1-S4 in the ESI $\dagger$ ), we studied $4 \mathrm{~T}$ using the faster CCSD and TDDFT methods. The choice of these methods allows no calculation of the SOC and we can only extrapolate from our knowledge of $2 \mathrm{~T}$ and $3 \mathrm{~T}$. The depopulation of the $\mathrm{S}_{1}$ state occurs via ISC as in the smaller oligothiophenes. The initial relaxation leads to the planar $\mathrm{S}_{1}$-Min-a, the $\mathrm{T}_{3}$ state intersects with the $\mathrm{S}_{1}$ state (see Table 6 and Table S16 (ESI $\dagger$ ) and Fig. 11) and should allow for the fast ISC channel (ISC1, Fig. 12). For the second ISC channel (ISC2) out of $\mathrm{S}_{1}$-Min-a to the $\mathrm{T}_{2}$ state torsional vibrational activity is again necessary to induce SOC between $T_{2}$ and $S_{1}$. For symmetry reasons the SOC between the $\mathrm{S}_{1}\left(B_{\mathrm{u}}\right)$ and the $\mathrm{T}_{3}\left(B_{\mathrm{u}}\right)$ is allowed at both the twisted $\mathrm{S}_{0}$-Min and the planar $\mathrm{S}_{1}$-Min-a geometries, while the SOC between the $\mathrm{S}_{1}\left(B_{\mathrm{u}}\right)$ and the $\mathrm{T}_{2}\left(A_{\mathrm{g}}\right)$ is allowed only for the twisted $\mathrm{S}_{0}$-Min.

Our conclusions are supported by the slow (390 ps) and fast (36 ps) time constants measured for the decay of the fluorescence of $4 \mathrm{~T} .{ }^{27}$ For comparison the fast time constant for this signal is found to be $16 \mathrm{ps}$ for $3 \mathrm{~T}$ while for the fast ESA decay and TTA rise it was 2 ps. The 390 ps time constant coincides well with the recently determined time constant for the TTA rise of 398 ps of $4 \mathrm{~T}^{40}{ }^{40}$ In addition a biexponential triplet formation was mentioned for $4 \mathrm{~T} .{ }^{31}$ We assume that the fast ISC channel of $4 \mathrm{~T}$ has not been observed until now as no femtosecond 
Table 6 Calculated CCSD vertical singlet and triplet excitation energies (eV) for the low-lying valence excited states of $4 \mathrm{~T}$ at the ground-state $\left(\mathrm{S}_{0}-\mathrm{Min}\right)$ and the $\mathrm{S}_{1}$ state $\left(\mathrm{S}_{1}-\mathrm{Min}-\mathrm{a}\right)$ minima. Oscillator strengths are shown in parentheses

\begin{tabular}{|c|c|c|c|c|c|c|}
\hline \multirow[b]{2}{*}{ State } & \multirow[b]{2}{*}{ Character } & \multicolumn{2}{|c|}{$\underline{\mathrm{S}_{0}-\operatorname{Min}\left(C_{2}\right)}$} & \multicolumn{3}{|c|}{$\underline{\mathrm{S}_{1} \text {-Min-a }\left(C_{2 \mathrm{~h}}\right)}$} \\
\hline & & Sym. & CCSD & Sym. & CCSD & Exp. \\
\hline $\mathrm{S}_{1}$ & $\pi_{1} \rightarrow \pi_{1}^{*}$ & $B$ & $4.00(1.30)$ & $B_{\mathrm{u}}$ & $3.30(1.40)$ & $2.59^{a}$ \\
\hline $\mathrm{S}_{2}$ & $\pi_{1} \rightarrow \pi_{2}^{*}$ & $A$ & $4.89(0.00)$ & $A_{\mathrm{g}}$ & $4.40(0.00)$ & - \\
\hline $\mathrm{T}_{1}$ & $\pi_{1} \rightarrow \pi_{1}^{*}$ & B & 2.36 & $B_{\mathrm{u}}$ & 1.59 & $1.75^{b}$ \\
\hline $\mathrm{T}_{2}$ & $\pi_{1} \rightarrow \pi_{2}^{*}$ & $A$ & 3.00 & $A_{\mathrm{g}}$ & 2.58 & $2.56^{b}$ \\
\hline $\mathrm{T}_{3}$ & $\pi_{1} \rightarrow \pi_{3}^{*}$ & $B$ & 3.64 & $B_{\mathrm{u}}$ & 3.44 & - \\
\hline $\mathrm{T}_{4}$ & $\pi_{2} \rightarrow \pi_{1}^{*}$ & $A$ & 4.16 & $A_{\mathrm{g}}$ & 4.14 & - \\
\hline
\end{tabular}

${ }^{a}$ Maximum of the fluorescence spectrum in dioxane at room temperature. ${ }^{25 b}$ Photodetachment photoelectron spectrum in the gas phase. ${ }^{47}$

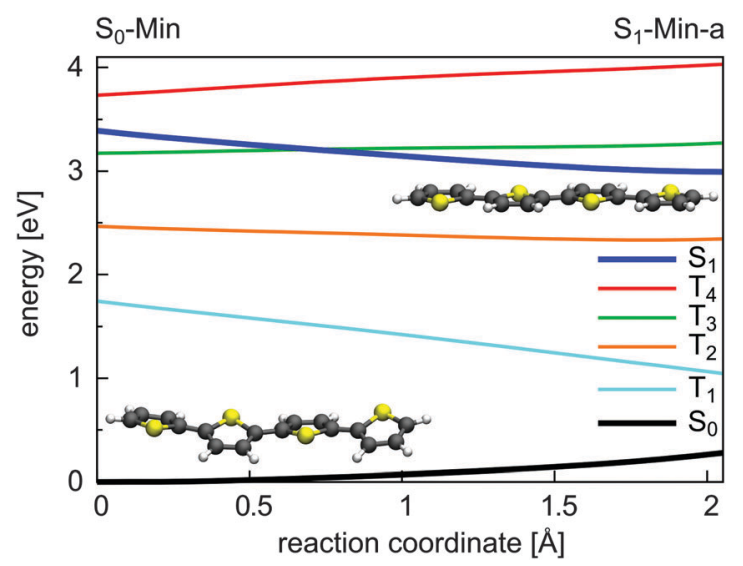

Fig. 11 Energy profile of the $S_{0}$, the $S_{1}$ and the four lowest lying triplet states along the $S_{1}$ relaxation coordinate of $4 \mathrm{~T}$. The energy calculations were performed at the TDDFT/6-31G* level of theory. The reaction coordinate was generated by linear interpolation between the optimized $\mathrm{S}_{0}-\mathrm{Min}\left(C_{2}\right.$ symmetry) and $\mathrm{S}_{1}$-Min-a $\left(C_{2 \mathrm{~h}}\right.$ symmetry) geometries.

time-resolved measurements with dependence on the excitation wavelength were performed similar to 3T. Based on the presented results we suggest that also in $4 \mathrm{~T}$ the fast ISC to the $\mathrm{T}_{3}$ state accounts for the high triplet quantum yield of $0.73 .^{25}$ Reasons for the decreasing triplet quantum yield from $2 \mathrm{~T}$ to $4 \mathrm{~T}$ are the diminishing SOC values along the initial relaxation (ISC1) and the increasing $\mathrm{S}_{1}-\mathrm{T}_{2}$ energy gap at $\mathrm{S}_{1}$-Min-a (ISC2) (see Table S18 in the ESI $\dagger$ ), also shown by photodetachment photoelectron spectroscopy. ${ }^{47}$

The return from the triplets back to the ground state involves similar steps as in $2 \mathrm{~T} . \mathrm{T}_{3}$ relaxation leads to its minimum where the outer thiophene rings are twisted versus the two planar inner rings (Fig. 12) due to the $\pi_{3}{ }^{*}$-orbital (Table 6) characterizing nonbonding and bonding interactions between the thiophene rings (see Fig. S23 in the ESI $\dagger$ ). The moderate $\mathrm{T}_{3}-\mathrm{T}_{2}$ energy gap of $0.41 \mathrm{eV}$ at $\mathrm{T}_{3}$-Min suggests the possibility of an internal conversion to $\mathrm{T}_{2}$. From there the $\mathrm{T}_{2}$ minimum can be reached characterized by an orthogonal arrangement of the thiophene rings with respect to the central bond (Fig. 12) due to the $\pi_{2}{ }^{*}$ orbital (see Fig. S23 in the ESI $\dagger$ ). At $\mathrm{T}_{2}$-Min the $\mathrm{T}_{2}$ and $\mathrm{T}_{1}$ states are degenerated like in $2 \mathrm{~T}$ (see Table S17 in ESI†), leading to fast

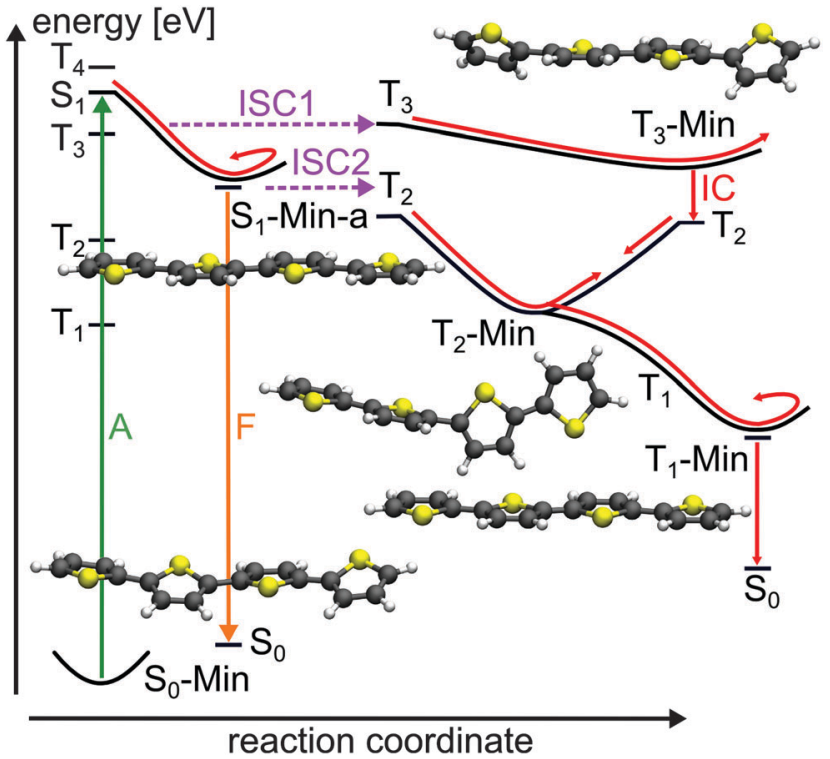

Fig. 12 Schematic illustration of the proposed deactivation mechanism of 4T after excitation to the $S_{1}$ state (A: absorption; $F$ : fluorescence; ISC: intersystem crossing; IC: internal conversion). The deactivation pathway is represented by red arrows. The relevant optimized geometries are shown as well.

internal conversion to $\mathrm{T}_{1}$. In $\mathrm{T}_{1}$ the global excited state minimum is reached, which is again planar (see Fig. 12). As no phosphorescence was detected, ${ }^{25}$ an ISC like in $2 \mathrm{~T}$ and $3 \mathrm{~T}$ should lead the molecule back to the ground state. The smaller $\mathrm{T}_{1}-\mathrm{S}_{0}$ energy gap of $1.0 \mathrm{eV}\left(\mathrm{CCSD} / 6-31 \mathrm{G}^{*}\right.$ level) at the $\mathrm{T}_{1}$-Min in comparison to $3 \mathrm{~T}(1.42 \mathrm{eV})$ and $2 \mathrm{~T}(1.77)$ could account for the decreasing triplet lifetime from $2 \mathrm{~T}$ to $4 \mathrm{~T}^{25}$ Although the fluorescence from $S_{1}$ is increased compared to $2 \mathrm{~T}$ and $3 \mathrm{~T},{ }^{25}$ the major part of the $4 \mathrm{~T}$ population still relaxes to the ground state via ISC and triplet states.

\section{Conclusions}

For thiophene $1 \mathrm{~T}$ and the oligothiophenes $2 \mathrm{~T}-4 \mathrm{~T}$ the relaxation processes from the first excited singlet state were investigated using quantum chemical calculations. We demonstrated that 1T decays primarily via its singlet states. Due to large singlettriplet energy gaps intersystem crossing (ISC) is ineffective, while internal conversion via a low lying $S_{1} / S_{0}$ conical intersection (CoIn) seam is associated with a very small barrier and thus highly efficient. In contrast the deactivation paths of the oligothiophenes are dominated by ISC. The $\mathrm{S}_{1} / \mathrm{S}_{0}$ CoIns are inactive although their energetic position with respect to the ground state minimum is similar for all systems. However, the excited $S_{1}$ state is significantly stabilized with increasing chain length due to its $\pi \pi^{*}$ character in the Franck-Condon (FC) region and at the $S_{1}$ minimum $\left(S_{1}\right.$-Min-a). At the $S_{1} / S_{0}$ CoIns the $\mathrm{S}_{1}$ state character has changed to $\pi \sigma^{*}$ which is more localized and does not profit to the same extent from the elongation of the $\pi$-system. Thus in the oligothiophenes the barriers towards the CoIns are increased and inhibit the passage through them. 
Furthermore the extension of the $\pi$-system induces more $\pi \pi^{*}$ states and shifts the triplet state $T_{3}$ below and close to the $S_{1}$ state at the FC point for all investigated oligothiophenes. During the initial relaxation from the non-planar conformation to the planar $S_{1}$-Min-a the $T_{3}$ state intersects with the $S_{1}$ state opening the first ISC path. Around $\mathrm{S}_{1}$-Min-a thermal torsional fluctuations can induce the second, less effective ISC channel with the $\mathrm{T}_{2}$ state. In view of the experimental findings and our results, we conclude that the first ISC channel is responsible for the high triplet quantum yields. Hereby we can say that the correlation between the planarization and the ISC is the key property. The overall diminishing SOC from $2 \mathrm{~T}$ to $4 \mathrm{~T}$ and the increasing $\mathrm{S}_{1}-\mathrm{T}_{2}$ energy gaps at $\mathrm{S}_{1}$-Min-a should account for the slightly decreasing triplet and increasing fluorescence quantum yields. ${ }^{25}$ A quantitative determination of the rate constants and branching ratios of the two ISC channels would require dynamical studies but these are clearly beyond the scope of the present work. The return from the triplets back to the ground state is made possible by inter-ring torsions and $T_{1} / S_{0}$ intersections, the latter are characterized by open-ring structures similar to the $S_{1} / S_{0}$ CoIns of $1 \mathrm{~T}$. We found two different kinds of triplet relaxation. For the even-numbered oligothiophenes we found $\mathrm{a}_{3} \rightarrow \mathrm{T}_{2} \rightarrow \mathrm{T}_{1}$ cascade, while for the odd oligothiophene an additional direct $\mathrm{T}_{3} \rightarrow \mathrm{T}_{1}$ path exists.

The present results in combination with previous theoretical and experimental observations offer a quite complete picture of the photophysics of $1 \mathrm{~T}-4 \mathrm{~T}$ and allow making predictions for the longer oligothiophenes. Also for the longer oligothiophenes and polythiophenes triplet formation has been observed. ${ }^{25,76-79}$ From our results for $2 \mathrm{~T}-4 \mathrm{~T}$ we extrapolate that the first ISC path should be present as well and non-planarity should enhance its efficiency. Preliminary calculations for 5T-10T show, with the exception of $6 \mathrm{~T}$, an intersection of the $\mathrm{S}_{1}$ state with a triplet state along the planarization to $\mathrm{S}_{1}$-Min-a (see Tables S19 and $\mathrm{S} 20$ in the ESI $\dagger$ ). The special behavior of $6 \mathrm{~T}$ is consistent with its highest fluorescence quantum yield $\left(\phi_{\mathrm{F}}\right)$ in the row from $2 \mathrm{~T}$ to $7 \mathrm{~T}^{25}$ Furthermore for polythiophenes in solution and films it was shown that $\phi_{\mathrm{F}}$ decreases due to more efficient nonradiative decay channels when the non-planarity of the thiophene backbone is increased by substitution. ${ }^{80}$ The higher degree of non-planarity should enhance the SOC and prolong the interaction time for the ISC. As triplet states are also present in polythiophene-based solar cells ${ }^{81,82}$ a deeper understanding of the relaxation processes and the factors influencing the efficiency of these processes should be a first step in improving the performance of the thiophene-based devices.

\section{Acknowledgements}

Financial support of this work from the Deutsche Forschungsgemeinschaft through the SFB749 and the excellence cluster 'Munich-Centre for Advanced Photonics' (MAP) is gratefully acknowledged. We thank Alexander André and Moritz Hönig for their contribution to the initial calculations of $1 \mathrm{~T}$ and $2 \mathrm{~T}$.

\section{References}

1 Handbook of Conducting Polymers, ed. T. A. Skotheim and J. R. Reynolds, CRC Press, Boca Raton, FL, 3rd edn, 2007.

2 Handbook of Thiophene-Based Materials: Applications in Organic Electronics and Photonics, ed. I. F. Perepichka and D. F. Perepichka, John Wiley \& Sons Ltd, Chichester, UK, 2007.

3 B. L. Rupert, W. J. Mitchell, A. J. Ferguson, M. E. Köse, W. L. Rance, G. Rumbles, D. S. Ginley, S. E. Shaheen and N. Kopidakis, J. Mater. Chem., 2009, 19, 5311-5324.

4 F. Zhang, D. Wu, Y. Xu and X. Feng, J. Mater. Chem., 2011, 21, 17590-17600.

5 G. Gigli, O. InganKäs, M. Anni, M. De Vittorio, R. Cingolani, G. Barbarella and L. Favaretto, Appl. Phys. Lett., 2001, 78, 1493-1495.

6 M. Mazzeo, D. Pisignano, L. Favaretto, G. Barbarella, R. Cingolani and G. Gigli, Synth. Met., 2003, 139, 671-673.

7 M. Irie, T. Fukaminato, K. Matsuda and S. Kobatake, Chem. Rev., 2014, 114, 12174-12277.

8 G. Barbarella, M. Zambianchi, A. Ventola, E. Fabiano, F. Della Sala, G. Gigli, M. Anni, A. Bolognesi, L. Polito, M. Naldi and M. Capobianco, Bioconjugate Chem., 2006, 17, 58-67.

9 H.-A. Ho, A. Najari and M. Leclerc, Acc. Chem. Res., 2008, 41, 168-178.

10 M. Zambianchi, F. D. Maria, A. Cazzato, G. Gigli, M. Piacenza, F. D. Sala and G. Barbarella, J. Am. Chem. Soc., 2009, 131, 10892-10900.

11 R. Håkansson, B. Nordén and E. W. Thulstrup, Chem. Phys. Lett., 1977, 50, 306-308.

12 D. Birnbaum and B. E. Kohler, J. Chem. Phys., 1989, 90, 3506-3510.

13 H. Chosrovian, S. Rentsch, D. Grebner, D. Dahm, E. Birckner and H. Naarmann, Synth. Met., 1993, 60, 23-26.

14 D. Lap, D. Grebner, S. Rentsch and H. Naarmann, Chem. Phys. Lett., 1993, 211, 135-139.

15 R. Rossi, M. Ciofalo and P. Glauco, J. Photochem. Photobiol., A, 1993, 70, 59-67.

16 S. Yamaguchi and H.-o. Hamaguchi, Chem. Phys. Lett., 1994, 227, 255-260.

17 J. E. Chadwick and B. E. Kohler, J. Phys. Chem., 1994, 98, 3631-3637.

18 W. J. Buma, B. E. Kohler and T. A. Shaler, J. Phys. Chem., 1994, 98, 4990-4992.

19 M. Belletête, M. Leclerc and G. Durocher, J. Phys. Chem., 1994, 98, 9450-9456.

20 R. Colditz, D. Grebner, M. Helbig and S. Rentsch, Chem. Phys., 1995, 201, 309-320.

21 R. S. Becker, J. Seixas de Melo, A. L. Maçanita and F. Elisei, Pure Appl. Chem., 1995, 67, 9-16.

22 D. Grebner, M. Helbig and S. Rentsch, J. Phys. Chem., 1995, 99, 16991-16998.

23 G. Lanzani, M. Nisoli, S. De Silvestri and R. Tubino, Chem. Phys. Lett., 1996, 251, 339-345.

24 P. Landwehr, H. Port and H. Wolf, Chem. Phys. Lett., 1996, 260, 125-129. 
25 R. S. Becker, J. Seixas de Melo, A. L. Maçanita and F. Elisei, J. Phys. Chem., 1996, 100, 18683-18695.

26 D. V. Lap, D. Grebner and S. Rentsch, J. Phys. Chem. A, 1997, 101, 107-112.

27 A. Yang, M. Kuroda, Y. Shiraishi and T. Kobayashi, J. Phys. Chem. B, 1998, 102, 3706-3711.

28 W. Paa, J.-P. Yang, M. Helbig, J. Hein and S. Rentsch, Chem. Phys. Lett., 1998, 292, 607-614.

29 N. DiCésare, M. Belletête, M. Leclerc and G. Durocher, Chem. Phys. Lett., 1998, 291, 487-495.

30 N. DiCésare, M. Belletête, C. Marrano, M. Leclerc and G. Durocher, J. Phys. Chem. A, 1999, 103, 795-802.

31 J. Yang, W. Paa and S. Rentsch, Synth. Met., 1999, 101, 624-625.

32 S. Rentsch, J. P. Yang, W. Paa, E. Birckner, J. Schiedt and R. Weinkauf, Phys. Chem. Chem. Phys., 1999, 1, 1707-1714.

33 J.-P. Yang, W. Paa and S. Rentsch, Chem. Phys. Lett., 2000, 320, 665-672.

34 W. Paa, J.-P. Yang and S. Rentsch, Appl. Phys. B: Lasers Opt., 2000, 71, 443-449.

35 W. Paa, J.-P. Yang and S. Rentsch, Synth. Met., 2001, 119, 525-526.

36 H. Haberkern, K. R. Asmis, M. Allan and P. Swiderek, Phys. Chem. Chem. Phys., 2003, 5, 827-833.

37 R. Weinkauf, L. Lehr, E. W. Schlag, S. Salzmann and C. M. Marian, Phys. Chem. Chem. Phys., 2008, 10, 393-404.

38 D. M. P. Holland, A. B. Trofimov, E. A. Seddon, E. V. Gromov, T. Korona, N. de Oliveira, L. E. Archer, D. Joyeux and L. Nahon, Phys. Chem. Chem. Phys., 2014, 16, 21629-21644.

39 J. Zhou, W. Yu and A. E. Bragg, J. Phys. Chem. Lett., 2015, 6, 3496-3502.

40 S.-m. Sun, S. Zhang, K. Liu, Y.-p. Wang and B. Zhang, Photochem. Photobiol. Sci., 2015, 14, 853-858.

41 S. Salzmann, M. Kleinschmidt, J. Tatchen, R. Weinkauf and C. M. Marian, Phys. Chem. Chem. Phys., 2008, 10, 380-392.

42 X.-F. Wu, X. Zheng, H.-G. Wang, Y.-Y. Zhao, X. Guan, D. L. Phillips, X. Chen and W. Fang, J. Chem. Phys., 2010, 133, 134507.

43 M. Stenrup, Chem. Phys., 2012, 397, 18-25.

44 G. Cui and W. Fang, J. Phys. Chem. A, 2011, 115, 11544-11550.

45 D. Fazzi, M. Barbatti and W. Thiel, Phys. Chem. Chem. Phys., 2015, 17, 7787-7799.

46 A. Prlj, B. F. E. Curchod and C. Corminboeuf, Phys. Chem. Chem. Phys., 2015, 17, 14719-14730.

47 S. Siegert, F. Vogeler, C. M. Marian and R. Weinkauf, Phys. Chem. Chem. Phys., 2011, 13, 10350-10363.

48 M. Rubio, M. Merchán, R. Pou-Amérigo and E. Ortí, ChemPhysChem, 2003, 4, 1308-1315.

49 S. H. Vosko, L. Wilk and M. Nusair, Can. J. Phys., 1980, 58, 1200-1211.

50 C. Lee, W. Yang and R. G. Parr, Phys. Rev. B: Condens. Matter Mater. Phys., 1988, 37, 785-789.

51 P. J. Stephens, F. J. Devlin, C. F. Chabalowski and M. J. Frisch, J. Phys. Chem., 1994, 98, 11623-11627.

52 A. D. Becke, J. Chem. Phys., 1993, 98, 5648-5652.
53 R. Krishnan, J. S. Binkley, R. Seeger and J. A. Pople, J. Chem. Phys., 1980, 72, 650-654.

54 H. A. Duarte, H. F. Dos Santos, W. R. Rocha and W. B. De Almeida, J. Chem. Phys., 2000, 113, 4206-4215.

55 P. Celani and H.-J. Werner, J. Chem. Phys., 2000, 112, 5546-5557.

56 J. F. Stanton and R. J. Bartlett, J. Chem. Phys., 1993, 98, 7029-7039.

57 T. Yanai, D. P. Tew and N. C. Handy, Chem. Phys. Lett., 2004, 393, 51-57.

58 M. M. Francl, W. J. Pietro, W. J. Hehre, J. S. Binkley, M. S. Gordon, D. J. DeFrees and J. A. Pople, J. Chem. Phys., 1982, 77, 3654-3665.

59 M. Rubio, M. Merchán and E. Ortí, ChemPhysChem, 2005, 6, 1357-1368.

60 M. Merchán, L. Serrano-Andrés, M. A. Robb and L. Blancafort, J. Am. Chem. Soc., 2005, 127, 1820-1825.

61 A. Berning, M. Schweizer, H.-J. Werner, P. J. Knowles and P. Palmieri, Mol. Phys., 2000, 98, 1823-1833.

62 H.-J. Werner, P. J. Knowles, G. Knizia, F. R. Manby and M. Schütz, Wiley Interdiscip. Rev.: Comput. Mol. Sci., 2012, 2, 242-253.

63 H.-J. Werner, P. J. Knowles, G. Knizia, F. R. Manby, M. Schütz, P. Celani, T. Korona, R. Lindh, A. Mitrushenkov, G. Rauhut, K. R. Shamasundar, T. B. Adler, R. D. Amos, A. Bernhardsson, A. Berning, D. L. Cooper, M. J. O. Deegan, A. J. Dobbyn, F. Eckert, E. Goll, C. Hampel, A. Hesselmann, G. Hetzer, T. Hrenar, G. Jansen, C. Köppl, Y. Liu, A. W. Lloyd, R. A. Mata, A. J. May, S. J. McNicholas, W. Meyer, M. E. Mura, A. Nicklass, D. P. O’Neill, P. Palmieri, D. Peng, K. Pflüger, R. Pitzer, M. Reiher, T. Shiozaki, H. Stoll, A. J. Stone, R. Tarroni, T. Thorsteinsson and M. Wang, MOLPRO, version 2012.1, a package of ab initio programs, 2012, see http://www.molpro.net/. 64 M. J. Frisch, G. W. Trucks, H. B. Schlegel, G. E. Scuseria, M. A. Robb, J. R. Cheeseman, G. Scalmani, V. Barone, B. Mennucci, G. A. Petersson, H. Nakatsuji, M. Caricato, X. Li, H. P. Hratchian, A. F. Izmaylov, J. Bloino, G. Zheng, J. L. Sonnenberg, M. Hada, M. Ehara, K. Toyota, R. Fukuda, J. Hasegawa, M. Ishida, T. Nakajima, Y. Honda, O. Kitao, H. Nakai, T. Vreven, J. A. Montgomery, Jr., J. E. Peralta, F. Ogliaro, M. Bearpark, J. J. Heyd, E. Brothers, K. N. Kudin, V. N. Staroverov, R. Kobayashi, J. Normand, K. Raghavachari, A. Rendell, J. C. Burant, S. S. Iyengar, J. Tomasi, M. Cossi, N. Rega, J. M. Millam, M. Klene, J. E. Knox, J. B. Cross, V. Bakken, C. Adamo, J. Jaramillo, R. Gomperts, R. E. Stratmann, O. Yazyev, A. J. Austin, R. Cammi, C. Pomelli, J. W. Ochterski, R. L. Martin, K. Morokuma, V. G. Zakrzewski, G. A. Voth, P. Salvador, J. J. Dannenberg, S. Dapprich, A. D. Daniels, Ö. Farkas, J. B. Foresman, J. V. Ortiz, J. Cioslowski and D. J. Fox, Gaussian 09, Revision D.01, Gaussian Inc., Wallingford, CT, 2009.

65 M. Ciofalo and G. L. Manna, Chem. Phys. Lett., 1996, 263, 73-78. 66 S. Millefiori, A. Alparone and A. Millefiori, J. Heterocycl. Chem., 2000, 37, 847-853.

67 F. Liu, P. Zuo, L. Meng and S. J. Zheng, THEOCHEM, 2005, 726, 161-169. 
68 G. Zerbi, B. Chierichetti and O. Ingänas, J. Chem. Phys., 1991, 94, 4637-4645.

69 D. Beljonne, Z. Shuai, G. Pourtois and J. L. Bredas, J. Phys. Chem. A, 2001, 105, 3899-3907.

70 M. Richter, P. Marquetand, J. González-Vázquez, I. Sola and L. González, J. Phys. Chem. Lett., 2012, 3, 3090-3095.

71 D. Parker, R. Minns, T. Penfold, G. Worth and H. Fielding, Chem. Phys. Lett., 2009, 469, 43-47.

72 T. Penfold and G. Worth, Chem. Phys., 2010, 375, 58-66.

73 M. Stenrup and A. Larson, Chem. Phys., 2011, 379, 6-12.

74 M. Breza, V. Lukeš and I. Vrábel, THEOCHEM, 2001, 572, 151-160.

75 W. Porzio, S. Destri, M. Mascherpa, S. Rossini and S. Brückner, Synth. Met., 1993, 55, 408-413.
76 B. Kraabel, D. Moses and A. J. Heeger, J. Chem. Phys., 1995, 103, 5102.

77 H. Burrows, J. Seixas de Melo, C. Serpa, L. Arnaut, M. G. Miguel, A. Monkman, I. Hamblett and S. Navaratnam, Chem. Phys., 2002, 285, 3-11.

78 S. Cook, A. Furube and R. Katoh, Energy Environ. Sci., 2008, 1, 294.

79 D. Sahoo, Y. Tian, G. Sforazzini, H. L. Anderson and I. G. Scheblykin, J. Mater. Chem. C, 2014, 2, 6601.

80 M. Theander, O. Inganäs, W. Mammo, T. Olinga, M. Svensson and M. R. Andersson, J. Phys. Chem. B, 1999, 103, 7771-7780.

81 Z. Xu and B. Hu, Adv. Funct. Mater., 2008, 18, 2611-2617.

82 M. Liedtke, A. Sperlich, H. Kraus, A. Baumann, C. Deibel, M. J. M. Wirix, J. Loos, C. M. Cardona and V. Dyakonov, J. Am. Chem. Soc., 2011, 133, 9088-9094. 